\title{
Exact non-reflecting boundary conditions revisited: well-posedness and stability
}

\author{
Sofia Eriksson ${ }^{a} \quad$ Jan Nordström ${ }^{b}$ \\ ${ }^{a}$ Department of Information Technology, Uppsala University, SE-75105 Uppsala, Sweden. \\ ${ }^{b}$ Department of Mathematics, Linköping University, SE-58183 Linköping, Sweden.
}

\begin{abstract}
Exact non-reflecting boundary conditions for an incompletely parabolic system have been studied. It is shown that well-posedness is a fundamental property of the non-reflecting boundary conditions. By using summation by parts operators for the numerical approximation and a weak boundary implementation, energy stability follows automatically. The stability in combination with the high order accuracy results in a reliable, efficient and accurate method. The theory is supported by numerical simulations.
\end{abstract}

\section{Introduction}

In computational fluid dynamics applications one often encounters the problem of how to limit the computational domain. For example, when simulating the flow field around an aircraft it is impossible to include the entire atmosphere, since that would be too computationally expensive. It is therefore necessary to truncate the domain at some distance away from the area of interest and introduce artificial boundary conditions $(\mathrm{ABC})$. Such boundaries will generate non-physical disturbances, and in many applications it is essential that these disturbances are minimized.

If the errors produced at the boundary stay localized, the boundary conditions have limited influence over the flow field and a simple boundary condition, e.g. the Dirichlet boundary condition could be used. However, this assumption is seldom valid, and when a wave encounters the boundary a significant portion will reflect back. This is illustrated in Figure 1(a), where the Dirichlet boundary condition pollutes the whole solution.

Apparently a better strategy is needed. In the classical paper [7], exact boundary closures are constructed in transformed space for the wave equation. Their approach is to express the solution as a superposition of waves, and eliminate the incoming waves at the boundaries. Similar techniques for deriving the non-reflecting boundary conditions (NRBC), for other types of equations, are used in $[15,11,17]$. Note that these conditions are exact, but formulated in transformed space.

Exact NRBC's are in most cases global in space and time, and can therefore be cumbersome to implement numerically. For that reason it is common to approximate 


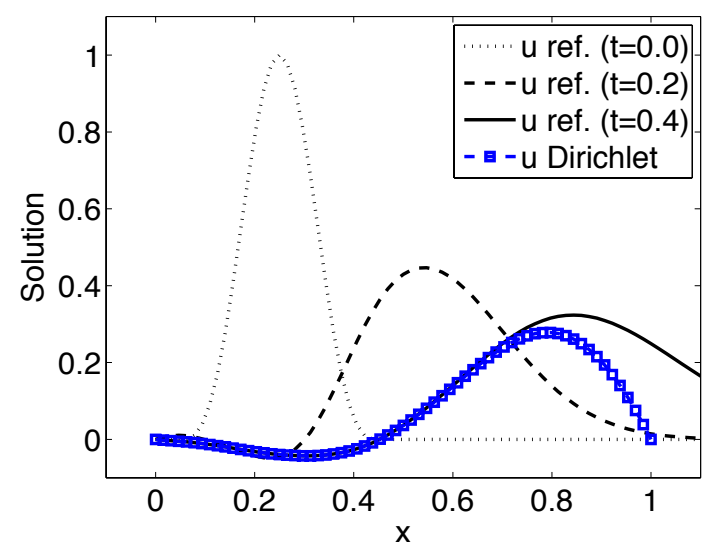

(a) Solution at $t=0.4$

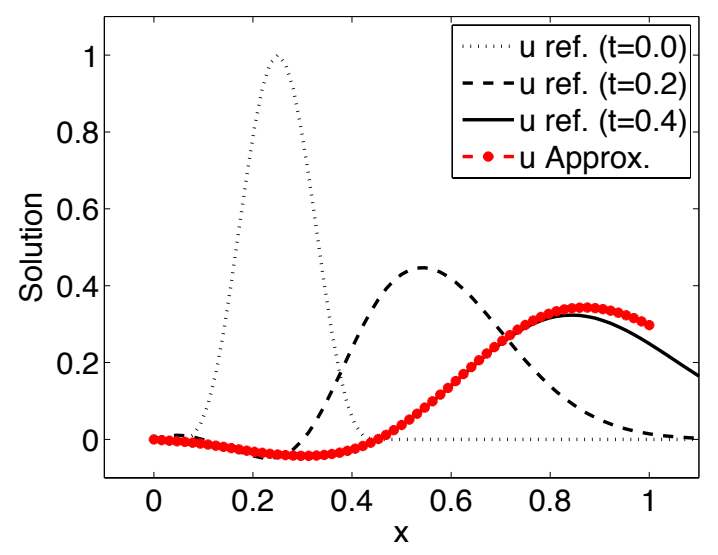

(b) Solution at $t=0.4$

Figure 1: The solution to equation (1), with initial condition given by (61). At $x=1$ the pulse should pass out without reflections. At the right boundary either a Dirichlet boundary condition or a zeroth order approximate NRBC is imposed.

or localize the NRBC's in space or time. In [7], where the exact NRBC's are made local in space and time using expansions, it is shown that some approximations are well-posed, and some ill-posed. To achieve boundary conditions that give sufficiently small reflections, high order expansions are necessary, which typically yields an ill-posed problem. For low order expansions, which result in a Dirichlet-to-Neumann map, it is easier to obtain well-posedness, and the results are still clearly better than the results obtained using the Dirichlet boundary condition, see Figure 1(b).

The main drawback with the approximative NRBC's are that they ruin the increased accuracy expected from mesh refinement of the interior scheme. From Table 1 it is evident that, although the solution obtained using the approximate NRBC's looks promising, it does not converge to the correct solution as we refine the mesh. There will always be an order one error remaining in the solution.

\begin{tabular}{|c|ccc|}
\hline$N$ & Error $(u)$ & ratio & conv. rate \\
\hline 16 & 0.01385376 & & \\
32 & 0.01407668 & 0.9842 & -0.0230 \\
64 & 0.01409021 & 0.9990 & -0.0014 \\
128 & 0.01409115 & 0.9999 & -0.0001 \\
\hline
\end{tabular}

Table 1: Results obtained using the approximative NRBC.

The area of ABC's has been the subject of massive research, see for example $[8$, $12,10,16,2]$, where the approach in $[16,2]$ yields local boundary conditions that can be made arbitrarily accurate. When it comes to the implementation of exact NRBC's it is, for special geometries, possible to localize the boundary conditions in time while still keeping them exact. This is exemplified in [9] where computations are performed for the wave equation on a spherical domain, and in [29] where highly accurate boundary conditions are used for a flow in a cylinder. See [15] for more details on exact and approximate NRBC on special computational domains. These techniques 
are unfortunately not always feasible, and in [22] computations are performed for the Schrödinger equation with the exact NRBC's using convolution quadratures. For an extensive review on ABC's, see [32]. An alternative to the above mentioned methods is to introduce buffer zones outside the artificial boundary, where the governing equations are modified such that waves are damped. When these zones are constructed to be exactly non-reflecting for the continuous problem, they are called perfectly matched layers (PML), see $[3,18,1]$.

In this paper we follow the work in [7] to some extent, but consider a slightly different problem and most importantly; no approximations will be used. Our main interest is the theoretical aspects of the problem, i.e. the well-posedness and stability properties of exact NRBC's. The exact boundary conditions are derived in the Laplace transformed space, and thereafter transformed back for the numerical simulations. The boundary conditions are hence global in time. We use high order accurate finite difference techniques, see $[25,4,31,27]$, such that the error originating from the interior discretization is kept at a minimum.

The core of this paper is that we show that the exact NRBC's result in a wellposed problem, and that this leads to energy estimates both for the continuous and the discrete formulation of the problem. We can thus, by a chain of arguments, guarantee a stable numerical procedure. The stability in combination with the high order accuracy results in a reliable, efficient and accurate method.

The paper is organized as follows. In section 2 we formulate the continuous problem. In section 3 exact non-reflecting boundary conditions are derived. In section 4 we show that the continuous problem is well-posed when using the non-reflecting boundary conditions, and that this leads to an energy estimate. The corresponding semi-discrete problem is presented in section 5. In section 6, two different approaches to choose the boundary procedure are presented, both leading to energy stability. Then, in section 7 , the boundary conditions which are derived in the Laplace transformed space are transformed to physical space using convolution quadratures. In section 8 numerical experiments are presented and conclusions are drawn in section 9.

\section{The continuous problem formulation}

Consider the linear $2 \times 2$ system of partial differential equations

$$
\begin{aligned}
& U_{t}+A U_{x}-B U_{x x}=F, \quad x \in\left[x_{L}, x_{R}\right], \quad t \geq 0 \\
& U=f, \quad x \in\left[x_{L}, x_{R}\right], \quad t=0 \\
& L_{L, R} U=g_{L, R}, \quad x=x_{L, R}, \quad t \geq 0,
\end{aligned}
$$

where

$$
U=\left[\begin{array}{l}
p \\
u
\end{array}\right], \quad A=\left[\begin{array}{ll}
v & c \\
c & v
\end{array}\right], \quad B=\left[\begin{array}{ll}
0 & 0 \\
0 & \varepsilon
\end{array}\right], \quad v>0 .
$$

$F(x, t)$ is the forcing function and $f(x)$ is the initial data. The operators $L_{L}$ and $L_{R}$ and the data $g_{L}$ and $g_{R}$ in the boundary conditions $L_{L, R} U=g_{L, R}$ are at this stage unknown. The Initial Boundary Value Problem (IBVP) (1) is incompletely parabolic and hence it has most of the properties and difficulties associated with the compressible 
Navier-Stokes equations. Throughout the paper we assume $v>0$. Exactly the same analysis can be done for negative values of $v$.

The Laplace transformed version of (1) is

$$
\begin{aligned}
s \hat{U}+A \hat{U}_{x}-B \hat{U}_{x x} & =\hat{F}+f, & & x \in\left[x_{L}, x_{R}\right] \\
\hat{L}_{L, R} \hat{U} & =\hat{g}_{L, R}, & & x=x_{L, R},
\end{aligned}
$$

where $s=\eta+\xi i$ is the dual variable to time, and where $\hat{U}=[\hat{p}, \hat{u}]^{T}$ is defined as

$$
\hat{U}(x, s)=\mathcal{L}\{U(x, t)\}=\int_{0}^{\infty} e^{-s t} U(x, t) d t, \quad \mathcal{L}\left\{U^{\prime}(x, t)\right\}=s \hat{U}(x, s)-U(x, 0) .
$$

To simplify the analysis, we write (2) on first order form by introducing $\hat{w}=\hat{u}_{x}$, which yields

$$
\begin{aligned}
\bar{S} \bar{U}+\bar{A} \bar{U}_{x} & =\bar{F}, & & x \in\left[x_{L}, x_{R}\right] \\
\bar{L}_{L, R} \bar{U} & =\hat{g}_{L, R}, & & x=x_{L, R},
\end{aligned}
$$

where $\bar{S}=\operatorname{diag}(s, s, 1)$ and where

$$
\bar{A}=\left[\begin{array}{ccc}
v & c & 0 \\
c & v & -\varepsilon \\
0 & -1 & 0
\end{array}\right], \quad \bar{U}=\left[\begin{array}{c}
\hat{p} \\
\hat{u} \\
\hat{w}
\end{array}\right], \quad \bar{F}=\left[\begin{array}{c}
\hat{F}_{1}+f_{1} \\
\hat{F}_{2}+f_{2} \\
0
\end{array}\right] .
$$

The solution to (3) consists of a homogenous and a particular part, such that $\bar{U}=$ $\bar{U}_{h}+\bar{U}_{p}$. The particular solution $\bar{U}_{p}$ (which depends on the data $\bar{F}$ ) is assumed to be known. The ansatz $\bar{U}_{h}=e^{\kappa x} \Psi$ leads to a generalized eigenvalue problem for $\kappa(s)$ and $\Psi(s)$ on the form

$$
(\bar{S}+\kappa \bar{A}) \Psi=0 .
$$

The eigenvalue problem (5) can only have non-trivial solutions $\Psi \neq 0$ if the determinant $|\bar{S}+\kappa \bar{A}|$ is zero. Written out explicitly the determinant is

$$
|\bar{S}+\kappa \bar{A}|=q(\kappa, s), \quad q(\kappa, s)=s^{2}+2 s v \kappa+\left(v^{2}-c^{2}-s \varepsilon\right) \kappa^{2}-\varepsilon v \kappa^{3} .
$$

Solving $q(\kappa, s)=0$ for the eigenvalues $\kappa$, and assuming that the three roots $\kappa_{j}$ are distinct, gives the general homogeneous solution

$$
\bar{U}_{h}=\sum_{j=1}^{3} \sigma_{j} e^{\kappa_{j} x} \Psi_{j}
$$

The coefficients $\sigma_{j}$ can be determined using the boundary conditions. This procedure is described in detail in $[13,26]$.

Remark: The solution $\bar{U}_{h}$ can be written on the form given in (7) unless $s=0$ at the same time as $v=c$, see Appendix A. In the rest of the paper we assume $v \neq c$. 


\section{Derivation of the boundary conditions}

Before the boundary conditions are constructed it is essential to know how many that are needed at each boundary. It is shown in [30] that for each negative $\operatorname{Re}(\kappa)$ we need one condition at the left boundary, and for each positive $\operatorname{Re}(\kappa)$ we need one condition at the right boundary. The number of roots with negative and positive real parts, respectively, is given by

Proposition 3.1. Consider the roots of $q(\kappa, s)=0$ in (6). For $v>0$ and s such that $\operatorname{Re}(s)>0$, two of the $\kappa$ 's have negative real part and one of the $\kappa$ 's has positive real part.

Proof. Assume that $\kappa$ passes the imaginary axis, i.e. that $\kappa=\beta i$. Inserting this into equation (6) and using that $s=\eta+\xi i$ yields

$$
c^{2} \beta^{2}+\varepsilon \eta \beta^{2}+\eta^{2}-(\xi+v \beta)^{2}+\left(2 \eta+\varepsilon \beta^{2}\right)(\xi+v \beta) i=0 .
$$

The imaginary part of (8) is zero if either $\xi+v \beta=0$ or $2 \eta+\varepsilon \beta^{2}=0$. In both of these cases, it is required that either $\eta<0$ or that $\eta=\xi=0$ to cancel the real part. That is, as long as the real part of $s$ is positive $(\eta>0)$, no purely imaginary $\kappa$ can exist and hence the real part of the $\kappa$ 's can not change sign. Dividing $q(\kappa, s)$ in $(6)$ by $-\varepsilon v$ yields

$$
\begin{gathered}
\tilde{q}(\kappa, s)=\kappa^{3}-\underbrace{\frac{\left(v^{2}-c^{2}-s \varepsilon\right)}{\varepsilon v}}_{r_{2}} \kappa^{2}+\underbrace{\frac{-2 s}{\varepsilon}}_{r_{1}} \kappa-\underbrace{\frac{s^{2}}{\varepsilon v}}_{r_{0}}=\left(\kappa-\kappa_{1}\right)\left(\kappa-\kappa_{2}\right)\left(\kappa-\kappa_{3}\right) \\
r_{2}=\kappa_{1}+\kappa_{2}+\kappa_{3}, \quad r_{1}=\kappa_{1} \kappa_{2}+\kappa_{1} \kappa_{3}+\kappa_{2} \kappa_{3}, \quad r_{0}=\kappa_{1} \kappa_{2} \kappa_{3},
\end{gathered}
$$

and by assuming $s$ real and large, we get $r_{0}>0, r_{1}<0$ and $r_{2}<0$. According to Descartes' rule of signs [28], the polynomial $\tilde{q}(\kappa, s)$ has exactly one positive root for these values of $r_{0}, r_{1}$ and $r_{2}$.

Thus two boundary conditions are needed at the left boundary and one boundary condition is needed at the right boundary. Without loss of generality, let $\operatorname{Re}\left(\kappa_{1}\right)<0$, $\operatorname{Re}\left(\kappa_{2}\right)<0$ and $\operatorname{Re}\left(\kappa_{3}\right)>0$. In addition, it holds that

Proposition 3.2. For $v>0$ and s such that $\operatorname{Re}(s)>0$, it holds that $\operatorname{Re}\left(\kappa_{1} / s\right)<0$, $\operatorname{Re}\left(\kappa_{2} / s\right)<0$ and $\operatorname{Re}\left(\kappa_{3} / s\right)>0$.

The proof of Proposition 3.2 is given in Appendix B.

\subsection{Non-reflecting boundary conditions}

One approach when constructing non-reflecting boundary conditions is to prohibit the solution outside the artificial boundary from growing, i.e. by demanding that $\bar{U}_{h}(x) \rightarrow 0$ as $x \rightarrow \pm \infty$, see [32]. This is accomplished by canceling the coefficients $\sigma_{j}$ in $(7)$ corresponding to the growing modes at each boundary.

Remark: Compare with the hyperbolic version of (1), where the characteristics of $U(x, t)$ travel with constant wave speed $a_{j}$. In this case the eigenvalues of the Laplace 
transformed solution have the form $\kappa_{j}=-s / a_{j}$ and the eigenvectors $\Psi_{j}$ are independent of $s$, such that

$$
U(x, t)=\sum_{j} h_{j}\left(t-x / a_{j}\right) \Psi_{j}, \quad \hat{U}(x, s)=\sum_{j} \hat{h}_{j}(s) e^{-x s / a_{j}} \Psi_{j} .
$$

Thus a positive wave speed $a_{j}$, which means that the eigensolution $\Psi_{j}$ is right-going, implies that $\operatorname{Re}\left(\kappa_{j}\right)=-\operatorname{Re}(s) / a_{j}$ is negative. Likewise, if $\operatorname{Re}\left(\kappa_{j}\right)>0$, the eigenfunction $\Psi_{j}$ is left-going. For a hyperbolic problem, providing zero data directly to the ingoing variables means that the outgoing waves can pass through the boundary freely, without reflections. Analogously, in (7) we should at each boundary cancel the modes that are growing outwards.

Recall that the real parts of $\kappa_{1}$ and $\kappa_{2}$ are negative and the real part of $\kappa_{3}$ is positive for $\operatorname{Re}(s)>0$. Our aim is to construct boundary conditions for the left boundary that force $\sigma_{1}$ and $\sigma_{2}$ to zero, or a boundary condition for the right boundary that forces $\sigma_{3}$ to zero. With access to the eigenvalues $\kappa_{i}$ we compute the eigenvectors $\Psi_{i}$ and the corresponding orthogonal vectors $\Phi_{i}$

$$
\Psi_{i}=\left[\begin{array}{c}
-c \\
\left(s+v \kappa_{i}\right) / \kappa_{i} \\
s+v k_{i}
\end{array}\right], \quad \Phi_{i}=\left[\begin{array}{c}
\varepsilon\left(v \kappa_{j}+s\right)\left(v \kappa_{k}+s\right) / s c \\
\varepsilon v \kappa_{j} \kappa_{k} / s \\
\varepsilon
\end{array}\right] .
$$

The vector $\Phi_{j}$ is orthogonal to $\Psi_{i}$ for $i \neq j$, such that

$$
\Phi_{i}^{T} \Psi_{i}=\varepsilon v\left(\kappa_{i}-\kappa_{j}\right)\left(\kappa_{i}-\kappa_{k}\right) / \kappa_{i}, \quad \Phi_{j}^{T} \Psi_{i}=0 .
$$

Using (7) and (11) we see that the boundary condition $\Phi_{i}^{T} \bar{U}_{h}=0$ is equivalent to $\sigma_{i} e^{\kappa_{i} x} \Phi_{i}^{T} \Psi_{i}=0$, which forces $\sigma_{i}$ to zero. This gives the exact non-reflecting boundary conditions

$$
x=x_{L}: \quad\left\{\begin{array}{l}
\Phi_{1}^{T} \bar{U}_{h}=0 \\
\Phi_{2}^{T} \bar{U}_{h}=0
\end{array}, \quad x=x_{R}: \quad \Phi_{3}^{T} \bar{U}_{h}=0 .\right.
$$

The boundary conditions (12) are $\bar{L}_{L} \bar{U}_{h}=0$ and $\bar{L}_{R} \bar{U}_{h}=0$, where

$$
\bar{L}_{L}=\left[\Phi_{1}, \Phi_{2}\right]^{T}, \quad \bar{L}_{R}=\Phi_{3}^{T} .
$$

Thus we can identify

$$
\bar{L}_{L, R} \bar{U}=\bar{L}_{L, R}\left(\bar{U}_{h}+\bar{U}_{p}\right)=\bar{L}_{L, R} \bar{U}_{p} \quad \Longrightarrow \quad \hat{g}_{L, R}=\bar{L}_{L, R} \bar{U}_{p} .
$$

Finding the data $\hat{g}_{L, R}$ can be difficult. Common choices are to assume that $\bar{U}_{p}$ is constant or zero. To take the possibility of non-exact data into account, assume that the boundary data has been chosen such that $\hat{g}_{L, R}=\bar{L}_{L, R} \bar{U}_{p}+g_{L, R}^{\prime}$. Then, in practice, the boundary conditions imposed are

$$
x=x_{L}: \quad \bar{L}_{L} \bar{U}_{h}=g_{L}^{\prime}, \quad x=x_{R}: \quad \bar{L}_{R} \bar{U}_{h}=g_{R}^{\prime},
$$

where $g_{L, R}^{\prime}$ should be some perturbation close to (or preferably equal to) zero.

Remark: The particular solution $\bar{U}_{p}$ depends on $\bar{F}$, which in turn depends on the forcing function $F$ and the initial function $f$ in (1). Often these functions are defined so that they have compact support, which implies that $\bar{U}_{p}=0$ at the boundaries and that $\hat{g}_{L, R}=0$. One reasonable exception is a constant non-zero background flow. 


\section{Well-posedness of the IBVP in the GKS sense}

The problem (1) is well-posed in the GKS ${ }^{1}$ sense if no solutions $U(x, t)$ that grow exponentially in time exist, see $[24,6,13,14,26]$. (A more generous definition of wellposedness, that opens up for a wider range of problems, is to accept bounded growth of the solution. In this paper we limit ourselves to zero growth.)

Remark: A problem is well-posed (Hadamard's well-posedness) if: i) A solution exists, ii) The solution is unique, iii) The solution depends continuously on provided data. We will assume that a unique solution of the problem (1) exists, and focus on the third requirement, which is equivalent to limit the growth of the solution, see [13].

Consider the homogeneous solution (7). By defining

$$
\Psi=\left[\Psi_{1}, \Psi_{2}, \Psi_{3}\right], \quad K(x)=\operatorname{diag}\left(e^{\kappa_{1} x}, e^{\kappa_{2} x}, e^{\kappa_{3} x}\right), \quad \sigma=\left[\sigma_{1}, \sigma_{2}, \sigma_{3}\right]^{T},
$$

we can write $\bar{U}_{h}=\Psi K \sigma$. Next, the boundary conditions in (14) are applied, yielding

$$
E(s) \sigma=g^{\prime}, \quad E(s)=\left[\begin{array}{c}
\bar{L}_{L} \Psi K\left(x_{L}\right) \\
\bar{L}_{R} \Psi K\left(x_{R}\right)
\end{array}\right]=\left[\begin{array}{ccc}
e^{\kappa_{1} x_{L}} \Phi_{1}^{T} \Psi_{1} & e^{\kappa_{2} x_{L}} \Phi_{1}^{T} \Psi_{2} & e^{\kappa_{3} x_{L}} \Phi_{1}^{T} \Psi_{3} \\
e^{\kappa_{1} x_{L}} \Phi_{2}^{T} \Psi_{1} & e^{\kappa_{2} x_{L}} \Phi_{2}^{T} \Psi_{2} & e^{\kappa_{3} x_{L}} \Phi_{2}^{T} \Psi_{3} \\
e^{\kappa_{1} x_{R}} \Phi_{3}^{T} \Psi_{1} & e^{\kappa_{2} x_{R}} \Phi_{3}^{T} \Psi_{2} & e^{\kappa_{3} x_{R}} \Phi_{3}^{T} \Psi_{3}
\end{array}\right],
$$

where $g^{\prime}=\left[\left(g_{L}^{\prime}\right)^{T},\left(g_{R}^{\prime}\right)^{T}\right]^{T}$. Each row of the system above corresponds to one boundary condition, and for general boundary conditions the matrix $E(s)$ is full. If $E(s)$ is non-singular we can solve for $\sigma$ and obtain a unique solution $\bar{U}=\bar{U}_{p}+\Psi K E(s)^{-1} g^{\prime}$. Recalling that the first two entries of $\bar{U}$ are denoted $\hat{U}$, we can formally transform back to the time domain, as

$$
U(x, t)=\mathcal{L}^{-1}\{\hat{U}\}=e^{\eta_{0} t}\left(\frac{1}{2 \pi} \int_{-\infty}^{+\infty} \hat{U}\left(x, \eta_{0}+i \xi\right) e^{i \xi t} d \xi\right)
$$

where $E(s)$ must be non-singular for $\eta>\eta_{0}$. The problem is well-posed in our restrictive GKS sense if $\eta_{0} \leq 0$, (for convergence to steady-state $\eta_{0}<0$ is necessary).

Proposition 4.1. Consider the ordinary differential equation (3) with boundary operators (13). The corresponding matrix $E(s)$ is non-singular for $\operatorname{Re}(s) \geq 0($ if $0<v \neq c)$, and hence the problem (1) is well-posed.

Proof. Using that $\Phi_{j}^{T} \Psi_{i}=0$ for $i \neq j$ leads to

$$
E(s)=\left[\begin{array}{ccc}
e^{\kappa_{1} x_{L}} \Phi_{1}^{T} \Psi_{1} & 0 & 0 \\
0 & e^{\kappa_{2} x_{L}} \Phi_{2}^{T} \Psi_{2} & 0 \\
0 & 0 & e^{\kappa_{3} x_{R}} \Phi_{3}^{T} \Psi_{3}
\end{array}\right] .
$$

From (11) we know that $\Phi_{i}^{T} \Psi_{i}=\varepsilon v\left(\kappa_{i}-\kappa_{j}\right)\left(\kappa_{i}-\kappa_{k}\right) / \kappa_{i}$ and thereby the three entries of $E(s)$ are non-zero if the roots $\kappa$ are distinct. In Appendix $\mathrm{A}$ it is shown that there are no multiple roots for $\operatorname{Re}(s) \geq 0$, unless $s=0$. This special case is treated separately in Appendix A.1, where it is shown that $\lim _{s \rightarrow 0} \Phi_{j}^{T} \Psi_{j} \neq 0$ as long as $v \neq c$. Consequently $|E(s)| \neq 0$ for all $\operatorname{Re}(s) \geq 0$ when $v \neq c$.

\footnotetext{
${ }^{1}$ GKS refers to the classical paper [14] by Gustafsson, Kreiss and Sundström.
} 


\subsection{Well-posedness in the GKS sense leads to energy stability}

Proposition 4.1 above shows that the exact non-reflecting boundary conditions yield well-posedness (in the GKS sense). Next we show that the non-reflecting boundary conditions also leads to an energy estimate.

Equation (2) is multiplied by the conjugate transpose of $\hat{U}$ (denoted $\hat{U}^{*}$ ) from the left and integrated with respect to $x$. Adding the complex conjugate of the resulting relation to itself, and using that $s=\eta+\xi i$, we get

$$
2 \eta \int_{x_{L}}^{x_{R}} \hat{U}^{*} \hat{U} d x+2 \int_{x_{L}}^{x_{R}} \hat{U}_{x}^{*} B \hat{U}_{x} d x=B T_{L}+B T_{R}
$$

where

$$
B T_{L}=\hat{U}^{*} A \hat{U}-\hat{U}^{*} B \hat{U}_{x}-\left.\hat{U}_{x}^{*} B \hat{U}\right|_{x_{L}}, \quad B T_{R}=-\hat{U}^{*} A \hat{U}+\hat{U}^{*} B \hat{U}_{x}+\left.\hat{U}_{x}^{*} B \hat{U}\right|_{x_{R}} .
$$

Note that the forcing term $\hat{F}+f$ is omitted since it does not affect well-posedness [13]. We know from the previous analysis of $E(s)$ that the operators in (13) give a well-posed problem. However, if the boundary conditions can be imposed such that the boundary terms $B T_{L}$ and $B T_{R}$ are non-positive we obtain an energy estimate, which will lead directly to stability for the discrete problem.

Since we have derived the boundary conditions for the first order form in (3) we rewrite (16) on the equivalent form

$$
B T_{L}=\left.\bar{U}^{*} \widetilde{A} \bar{U}\right|_{x_{L}}, \quad B T_{R}=-\left.\bar{U}^{*} \widetilde{A} \bar{U}\right|_{x_{R}}, \quad \widetilde{A}=\left[\begin{array}{ccc}
v & c & 0 \\
c & v & -\varepsilon \\
0 & -\varepsilon & 0
\end{array}\right] .
$$

Remark: Well-posedness in the GKS sense considers the homogenous solution, $\bar{U}_{h}=$ $\sum_{j} \sigma_{j} e^{\kappa_{j} x} \Psi_{j}$. Computing the energy estimate for the homogenous solution instead of the total solution only affects the forcing term (which is disregarded) and hence the boundary terms in (17) holds for $\bar{U}_{h}$ as well as for $\bar{U}$.

Proposition 4.2. The left boundary term in (17) is non-positive, i.e. $B T_{L} \leq 0$.

Proof. The left boundary conditions in (12) force $\sigma_{1}$ and $\sigma_{2}$ to zero which yields the solution $\bar{U}_{h}=\sigma_{3} e^{\kappa_{3} x} \Psi_{3}$. Inserting this into $B T_{L}$ in (17) we obtain

$$
B T_{L}=\left|\sigma_{3} e^{\kappa_{3} x_{L}}\right|^{2} \mathcal{A}_{L}
$$

where

$$
\mathcal{A}_{L}=\Psi_{3}^{*} \widetilde{A} \Psi_{3}=-\operatorname{Re}\left(\frac{s}{\kappa_{3}}\right)\left(c^{2}+\left|\frac{s+v \kappa_{3}}{\kappa_{3}}\right|^{2}\right)-\varepsilon \operatorname{Re}\left(\kappa_{3}\right)\left|\frac{s+v \kappa_{3}}{\kappa_{3}}\right|^{2} \leq 0 .
$$

In the last inequality we used that $\operatorname{Re}\left(\kappa_{3} / s\right)>0$ and $\operatorname{Re}\left(\kappa_{3}\right)>0$ for $\operatorname{Re}(s)>0$, see Proposition 3.1 and Proposition 3.2.

Proposition 4.3. The right boundary term in (17) is non-positive, i.e. $B T_{R} \leq 0$. 
The proof of Proposition 4.3 is given in Appendix C.1 and results in

$$
B T_{R}=-\left[\begin{array}{l}
\sigma_{1} e^{\kappa_{1} x_{R}} \\
\sigma_{2} e^{\kappa_{2} x_{R}}
\end{array}\right]^{*} \mathcal{A}_{R}\left[\begin{array}{l}
\sigma_{1} e^{\kappa_{1} x_{R}} \\
\sigma_{2} e^{\kappa_{2} x_{R}}
\end{array}\right]
$$

where

$$
\mathcal{A}_{R}=\left[\begin{array}{ll}
\Psi_{1}^{*} \widetilde{A} \Psi_{1} & \Psi_{1}^{*} \widetilde{A} \Psi_{2} \\
\Psi_{2}^{*} \widetilde{A} \Psi_{1} & \Psi_{2}^{*} \widetilde{A} \Psi_{2}
\end{array}\right] \geq 0
$$

Since the boundary terms $B T_{L}$ and $B T_{R}$ are negative the right hand side of (15) is bounded, which leads to an energy estimate.

In the proofs of Proposition 4.2 and Proposition 4.3 we have assumed that the provided data is exact, such that $\sigma_{1,2}=0$ at the left boundary or $\sigma_{3}=0$ at the right boundary. Later in Section 6 we will also include the possibility of having non-zero (uncorrect) boundary data and show that the problem is in fact strongly well-posed.

\section{The semi-discrete problem formulation}

The boundary operators in (13) can be written

$$
\bar{L}_{L}=\left[\begin{array}{ccc}
\alpha_{1} & \beta_{1} & \varepsilon \\
\alpha_{2} & \beta_{2} & \varepsilon
\end{array}\right], \quad \hat{g}_{L}=\left[\begin{array}{c}
\hat{g}_{1} \\
\hat{g}_{2}
\end{array}\right], \quad \bar{L}_{R}=\left[\begin{array}{lll}
\alpha_{3} & \beta_{3} & \varepsilon
\end{array}\right], \quad \hat{g}_{R}=\left[\hat{g}_{3}\right],
$$

where $\alpha_{j}, \beta_{j}$ depend on $s$ and $\kappa_{j}(s)$. The structure of the complementing vectors in (10) and the relations in (9) gives

$$
\alpha_{i}=\frac{-s c}{s+v \kappa_{i}}, \quad \quad \beta_{i}=\frac{s}{\kappa_{i}} .
$$

The boundary conditions can also be rewritten such that they are appropriate for the problem (2), as

$$
\begin{aligned}
& \hat{L}_{L} \hat{U}=H_{L} \hat{U}+G_{L} \hat{U}_{x}=\hat{g}_{L} \Longleftrightarrow\left[\begin{array}{ll}
\alpha_{1} & \beta_{1} \\
\alpha_{2} & \beta_{2}
\end{array}\right]\left[\begin{array}{l}
\hat{p} \\
\hat{u}
\end{array}\right]+\left[\begin{array}{ll}
0 & \varepsilon \\
0 & \varepsilon
\end{array}\right]\left[\begin{array}{l}
\hat{p}_{x} \\
\hat{u}_{x}
\end{array}\right]=\left[\begin{array}{l}
\hat{g}_{1} \\
\hat{g}_{2}
\end{array}\right] \\
& \hat{L}_{R} \hat{U}=H_{R} \hat{U}+G_{R} \hat{U}_{x}=\hat{g}_{R} \Longleftrightarrow\left[\begin{array}{ll}
\alpha_{3} & \beta_{3}
\end{array}\right]\left[\begin{array}{l}
\hat{p} \\
\hat{u}
\end{array}\right]+\left[\begin{array}{ll}
0 & \varepsilon
\end{array}\right]\left[\begin{array}{l}
\hat{p}_{x} \\
\hat{u}_{x}
\end{array}\right]=\left[\hat{g}_{3}\right] .
\end{aligned}
$$

\subsection{The numerical scheme}

The domain $x \in\left[x_{L}, x_{R}\right]$ is discretized in space using $N+1$ equidistant grid points, as $x_{i}=x_{L}+\left(x_{R}-x_{L}\right) i / N$, where $i=0,1, \ldots, N$. The solution $U$ is represented by the discrete solution vector $V$ such that

$$
V=\left[V_{0}^{T}, V_{1}^{T}, \ldots, V_{N}^{T}\right]^{T}, \quad V_{i}(t) \approx U\left(x_{i}, t\right) .
$$

The semi-discrete scheme representing the IBVP in (1) is written

$$
\begin{aligned}
V_{t}+(D \otimes A) V-\left(D^{2} \otimes B\right) V & =F+\left(\left(\Sigma_{0} * V\right)(t)-\Gamma_{0}\right)+\left(\left(\Sigma_{N} * V\right)(t)-\Gamma_{N}\right), \\
V(0) & =f,
\end{aligned}
$$


where the symbol $\otimes$ refers to the Kronecker product. The boundary conditions are imposed weakly using the Simultaneous Approximation Term (SAT) technique, by the penalty terms $\left(\left(\Sigma_{0, N} * V\right)(t)-\Gamma_{0, N}(t)\right)$ which are yet unknown but will be derived in the Laplace transformed domain. Further, the difference operator $D$ (which mimics $\partial / \partial x$ ) is on so called Summation-By-Parts (SBP) form, and hence the following holds

$$
D=P^{-1} Q, \quad Q+Q^{T}=e_{N} e_{N}^{T}-e_{0} e_{0}^{T}, \quad P=P^{T}>0,
$$

where $e_{0}=[1,0, \ldots, 0]^{T}$ and $e_{N}=[0, \ldots, 0,1]^{T}$. The second derivative $\partial^{2} / \partial x^{2}$ is approximated by the wide operator $D^{2}$. For a read-up on SBP and SAT, see [23, 5] and references therein. Note that we use the same notation for $F, f$ both in the continuous and the discrete setting.

By Laplace transforming (23) the discrete representation of (2) is obtained, as

$$
s \hat{V}+(D \otimes A) \hat{V}-\left(D^{2} \otimes B\right) \hat{V}=\hat{F}+f+\left(\hat{\Sigma}_{0} \hat{V}-\hat{\Gamma}_{0}\right)+\left(\hat{\Sigma}_{N} \hat{V}-\hat{\Gamma}_{N}\right),
$$

where $\hat{V}(s)=\mathcal{L}\{V(t)\}$ and where $\hat{\Sigma}_{0, N}, \hat{\Gamma}_{0, N}$ remains to be determined. As in the continuous case we simplify by omitting the forcing function $\hat{F}+f$. We multiply (25) by $\hat{V}^{*} \bar{P}$ from the left, where $\bar{P}=P \otimes I_{2}$, and add the conjugate transpose of the equation to itself. Thereafter using the SBP-properties in (24) yields the energy estimate

$$
2 \eta \hat{V}^{*} \bar{P} \hat{V}+2(\bar{D} \hat{V})^{*}(P \otimes B) \bar{D} \hat{V}=B T_{L}^{D}+B T_{R}^{D},
$$

where $\bar{D}=D \otimes I_{2}$ and where

$$
\begin{aligned}
& B T_{L}^{D}=\hat{V}_{0}^{*} A \hat{V}_{0}-\hat{V}_{0}^{*} B(\bar{D} \hat{V})_{0}-(\bar{D} \hat{V})_{0}^{*} B \hat{V}_{0}+\hat{V}^{*} \bar{P}\left(\hat{\Sigma}_{0} \hat{V}-\hat{\Gamma}_{0}\right)+\left(\hat{\Sigma}_{0} \hat{V}-\hat{\Gamma}_{0}\right)^{*} \bar{P} \hat{V} \\
& B T_{R}^{D}=-\hat{V}_{N}^{*} A \hat{V}_{N}+\hat{V}_{N}^{*} B(\bar{D} \hat{V})_{N}+(\bar{D} \hat{V})_{N}^{*} B \hat{V}_{N}+\hat{V}^{*} \bar{P}\left(\hat{\Sigma}_{N} \hat{V}-\hat{\Gamma}_{N}\right)+\left(\hat{\Sigma}_{N} \hat{V}-\hat{\Gamma}_{N}\right)^{*} \bar{P} \hat{V} .
\end{aligned}
$$

Note the similarity between the semi-discrete energy estimate (26) and the continuous one in (15).

The matrices $\hat{\Sigma}_{0, N}$ and the vectors $\hat{\Gamma}_{0, N}$ depend on how the boundary conditions are imposed. We use the following ansätze for the penalty terms

$$
\begin{aligned}
& \hat{\Sigma}_{0} \hat{V}-\hat{\Gamma}_{0}=\left(P^{-1} e_{0} \otimes \tau_{0}+P^{-1} D^{T} e_{0} \otimes \sigma_{0}\right)\left(H_{L} \hat{V}_{0}+G_{L}(\bar{D} \hat{V})_{0}-\hat{g}_{L}\right) \\
& \hat{\Sigma}_{N} \hat{V}-\hat{\Gamma}_{N}=\left(P^{-1} e_{N} \otimes \tau_{N}+P^{-1} D^{T} e_{N} \otimes \sigma_{N}\right)\left(H_{R} \hat{V}_{N}+G_{R}(\bar{D} \hat{V})_{N}-\hat{g}_{R}\right),
\end{aligned}
$$

where all dependence of boundary data sits in $\hat{\Gamma}_{0, N}$, such that $\hat{\Gamma}_{0, N}=0$ if $\hat{g}_{L, R}=0$. The boundary operators $H_{L, R}, G_{L, R}$ are given in (22) and the penalty parameters $\tau_{0}$ and $\sigma_{0}$ are $2 \times 2$ matrices and $\tau_{N}$ and $\sigma_{N}$ are $2 \times 1$ vectors. The relations in (28) lead to

$$
\begin{aligned}
\hat{V}^{*} \bar{P}\left(\hat{\Sigma}_{0} \hat{V}-\hat{\Gamma}_{0}\right) & =\left(\hat{V}_{0}^{*} \tau_{0}+(\bar{D} \hat{V})_{0}^{*} \sigma_{0}\right)\left(H_{L} \hat{V}_{0}+G_{L}(\bar{D} \hat{V})_{0}-\hat{g}_{L}\right) \\
\hat{V}^{*} \bar{P}\left(\hat{\Sigma}_{N} \hat{V}-\hat{\Gamma}_{N}\right) & =\left(\hat{V}_{N}^{*} \tau_{N}+(\bar{D} \hat{V})_{N}^{*} \sigma_{N}\right)\left(H_{R} \hat{V}_{N}+G_{R}(\bar{D} \hat{V})_{N}-\hat{g}_{R}\right) .
\end{aligned}
$$

Inserting the expressions (29) into (27), the boundary terms can be written as

$$
\begin{aligned}
B T_{L}^{D} & =\left[\begin{array}{c}
\hat{V}_{0} \\
(\bar{D} \hat{V})_{0}
\end{array}\right]^{*}\left[\begin{array}{cc}
A+\tau_{0} H_{L}+\left(\tau_{0} H_{L}\right)^{*} & -B+\tau_{0} G_{L}+\left(\sigma_{0} H_{L}\right)^{*} \\
-B+\sigma_{0} H_{L}+\left(\tau_{0} G_{L}\right)^{*} & \sigma_{0} G_{L}+\left(\sigma_{0} G_{L}\right)^{*}
\end{array}\right]\left[\begin{array}{c}
\hat{V}_{0} \\
(\bar{D} \hat{V})_{0}
\end{array}\right] \\
& -\left[\begin{array}{c}
\hat{V}_{0} \\
(\bar{D} \hat{V})_{0}
\end{array}\right]^{*}\left[\begin{array}{c}
\tau_{0} \\
\sigma_{0}
\end{array}\right] \hat{g}_{L}-\left(\left[\begin{array}{c}
\hat{V}_{0} \\
(\bar{D} \hat{V})_{0}
\end{array}\right]^{*}\left[\begin{array}{c}
\tau_{0} \\
\sigma_{0}
\end{array}\right] \hat{g}_{L}\right)^{*}
\end{aligned}
$$


and

$$
\begin{aligned}
B T_{R}^{D} & =\left[\begin{array}{c}
\hat{V}_{N} \\
(\bar{D} \hat{V})_{N}
\end{array}\right]^{*}\left[\begin{array}{cc}
-A+\tau_{N} H_{R}+\left(\tau_{N} H_{R}\right)^{*} & B+\tau_{N} G_{R}+\left(\sigma_{N} H_{R}\right)^{*} \\
B+\sigma_{N} H_{R}+\left(\tau_{N} G_{R}\right)^{*} & \sigma_{N} G_{R}+\left(\sigma_{N} G_{R}\right)^{*}
\end{array}\right]\left[\begin{array}{c}
\hat{V}_{N} \\
(\bar{D} \hat{V})_{N}
\end{array}\right] \\
& -\left[\begin{array}{c}
\hat{V}_{N} \\
(\bar{D} \hat{V})_{N}
\end{array}\right]^{*}\left[\begin{array}{c}
\tau_{N} \\
\sigma_{N}
\end{array}\right] \hat{g}_{R}-\left(\left[\begin{array}{c}
\hat{V}_{N} \\
(\bar{D} \hat{V})_{N}
\end{array}\right]^{*}\left[\begin{array}{c}
\tau_{N} \\
\sigma_{N}
\end{array}\right] \hat{g}_{R}\right)^{*},
\end{aligned}
$$

respectively.

Similarly to the definition of well-posedness for the continuous problem, a numerical scheme is energy stable if the growth of the solution is bounded. As in the continuous case we limit ourselves to zero growth, which means that $\hat{V}^{*} \bar{P} \hat{V} \leq 0$ in (26) is needed. Hence, to prove stability, we must show that the boundary terms in (30) and (31) are non-positive for zero data. In the next section, we present two distinctly different ways of choosing the penalty parameters $\tau_{0, N}$ and $\sigma_{0, N}$ to achieve $B T_{L, R}^{D} \leq 0$.

\section{$6 \quad$ Energy estimates in Laplace space}

The stability requirements alone do not determine the penalty parameters $\tau_{0, N}$ and $\sigma_{0, N}$ in (28) uniquely. We will here present two different possible choices (here referred to as "replacing the indefinite terms" and "replacing the ingoing variables"), both guaranteeing a stable numerical scheme. In both cases the strategy is to first reformulate the continuous boundary terms $B T_{L, R}$ using the boundary conditions, and then to choose the penalty parameters such that the discrete boundary terms $B T_{L, R}^{D}$ mimic the continuous ones.

\subsection{Replacing the indefinite terms}

\subsubsection{The continuous formulation}

When inserting the boundary conditions (22) into (16), a more convenient form of the boundary conditions would be

$$
\begin{aligned}
\widetilde{H}_{L} \hat{U}+B \hat{U}_{x} & =\tilde{g}_{L}, & & x=x_{L} \\
\widetilde{H}_{R} \hat{U}+B \hat{U}_{x} & =\tilde{g}_{R}, & & x=x_{R} .
\end{aligned}
$$

To achieve this form, we construct two scaling matrices $S_{L}$ and $S_{R}$ such that $B=S_{L} G_{L}$ and $B=S_{R} G_{R}$. The scaling matrices are

$$
S_{L}=\left[\begin{array}{cc}
a & -a \\
b & 1-b
\end{array}\right], \quad S_{R}=\left[\begin{array}{l}
0 \\
1
\end{array}\right]
$$

where $a \neq 0$ and $b$ are arbitrary since $G_{L}$ is singular. Multiplying the original boundary conditions (22) by the scaling matrices yields (32) where $\widetilde{H}_{L}=S_{L} H_{L}, \tilde{g}_{L}=S_{L} \hat{g}_{L}$ and 
$\widetilde{H}_{R}=S_{R} H_{R}, \tilde{g}_{R}=S_{R} \hat{g}_{R}$. Written out explicitly, the boundary conditions in (32) are

$$
\begin{gathered}
\underbrace{\left[\begin{array}{cc}
a \Delta_{\alpha} & a \Delta_{\beta} \\
b \Delta_{\alpha}+\alpha_{2} & b \Delta_{\beta}+\beta_{2}
\end{array}\right]}_{\widetilde{H}_{L}}\left[\begin{array}{l}
\hat{p} \\
\hat{u}
\end{array}\right]+\underbrace{\left[\begin{array}{ll}
0 & 0 \\
0 & \varepsilon
\end{array}\right]}_{B}\left[\begin{array}{c}
\hat{p}_{x} \\
\hat{u}_{x}
\end{array}\right]=\underbrace{\left[\begin{array}{c}
a\left(\hat{g}_{1}-\hat{g}_{2}\right) \\
b\left(\hat{g}_{1}-\hat{g}_{2}\right)+\hat{g}_{2}
\end{array}\right]}_{\tilde{g}_{L}} \\
\underbrace{\left[\begin{array}{cc}
0 & 0 \\
\alpha_{3} & \beta_{3}
\end{array}\right]}_{\widetilde{H}_{R}}\left[\begin{array}{c}
\hat{p} \\
\hat{u}
\end{array}\right]+\underbrace{\left[\begin{array}{ll}
0 & 0 \\
0 & \varepsilon
\end{array}\right]}_{B}\left[\begin{array}{c}
\hat{p}_{x} \\
\hat{u}_{x}
\end{array}\right]=\underbrace{\left[\begin{array}{c}
0 \\
\hat{g}_{3}
\end{array}\right]}_{\tilde{g}_{R}},
\end{gathered}
$$

where we have defined $\Delta_{\beta}=\beta_{1}-\beta_{2}$ and $\Delta_{\alpha}=\alpha_{1}-\alpha_{2}$. When replacing the terms containing $B \hat{U}_{x}$ in (16) by the ones in (32) the boundary terms become

$$
\begin{aligned}
& B T_{L}=\hat{U}^{*} \underbrace{\left(A+\widetilde{H}_{L}+\widetilde{H}_{L}^{*}\right)}_{M_{L}} \hat{U}-\hat{U}^{*} \tilde{g}_{L}-\left.\tilde{g}_{L}^{*} \hat{U}\right|_{x_{L}} \\
& B T_{R}=-\hat{U}^{*} \underbrace{\left(A+\widetilde{H}_{R}+\widetilde{H}_{R}^{*}\right)}_{M_{R}} \hat{U}+\hat{U}^{*} \tilde{g}_{R}+\left.\tilde{g}_{R}^{*} \hat{U}\right|_{x_{R}} .
\end{aligned}
$$

For an energy estimate we need $M_{L}=A+\widetilde{H}_{L}+\widetilde{H}_{L}^{*}$ in (34) to be negative semi-definite and $M_{R}=A+\widetilde{H}_{R}+\widetilde{H}_{R}^{*}$ to be positive semi-definite.

Proposition 6.1. If the constants $a \neq 0$ and $b$ in $S_{L}$ in (33) satisfy

$$
\operatorname{Re}\left(a \Delta_{\alpha}+b \Delta_{\beta}\right) \leq \frac{\left|\Delta_{\alpha}\right|^{2}+\left|\Delta_{\beta}\right|^{2}}{2 r\left|\Delta_{\alpha}\right|^{2}}\left(\left|a \Delta_{\beta}^{*}-b \Delta_{\alpha}^{*}+\mu^{*}\right|^{2}-v^{2}-2 v \operatorname{Re}\left(\beta_{2}\right)+\left|c+\alpha_{2}\right|^{2}-|\mu|^{2}\right),
$$

where

$$
\begin{aligned}
\mu & =\frac{-2 \operatorname{Re}\left(\beta_{2}\right) \Delta_{\alpha} \Delta_{\beta}+\left(c+\alpha_{2}\right) \Delta_{\beta}^{2}-\left(c+\alpha_{2}\right)^{*} \Delta_{\alpha}^{2}}{\left|\Delta_{\alpha}\right|^{2}+\left|\Delta_{\beta}\right|^{2}} \\
r & =v+2 \operatorname{Re}\left(\beta_{2}-\left(c+\alpha_{2}\right) \frac{\Delta_{\beta}}{\Delta_{\alpha}}\right)+v\left|\frac{\Delta_{\beta}}{\Delta_{\alpha}}\right|^{2},
\end{aligned}
$$

then $M_{L}=A+\widetilde{H}_{L}+\widetilde{H}_{L}^{*}$ in (34) is negative semi-definite.

The proof of Proposition 6.1 is given in Appendix C.2.

Remark: At the left boundary we have three variables and two boundary conditions, hence it is possible to write the boundary term expressed in one variable only. Using the $M_{L}$ form in (34) this is disregarded and the boundary term is expressed as a combination of two variables, $\hat{p}$ and $\hat{u}$, even though these variables have an interrelation. So, although the choice of $a$ and $b$ has no impact on the well-posedness of the continuous problem, the definiteness of $M_{L}$ is crucial if we consider a solution that does not fulfill the boundary conditions exactly (i.e. a numerical solution). We will come back to this in the numerical sections.

Proposition 6.2. The matrix $M_{R}=A+\widetilde{H}_{R}+\widetilde{H}_{R}^{*}$ in (34) is positive semi-definite. 
Proof. Written out explicitly, we have

$$
M_{R}=\left[\begin{array}{cc}
v & c+\alpha_{3}^{*} \\
c+\alpha_{3} & v+\beta_{3}+\beta_{3}^{*}
\end{array}\right],
$$

and it is possible to show that $M_{R}=Z^{*} \mathcal{A}_{R} Z$, where

$$
Z=\left[\begin{array}{cc}
-c & -c \\
s / \kappa_{1}+v & s / \kappa_{2}+v
\end{array}\right]^{-1} .
$$

According to Sylvester's law of inertia, $M_{R} \geq 0$, since $\mathcal{A}_{R} \geq 0$ in (19).

Remark: By showing that $M_{L} \leq 0$ and $M_{R} \geq 0$ we do not only achieve an energy estimate for the assumption $\hat{g}_{L, R}=0$, but actually the solution is bounded also if using arbitrary data. The equations in (34) can easily be rewritten as

$$
\begin{aligned}
& B T_{L}=\left(\hat{U}-M_{L}^{-1} \tilde{g}_{L}\right)^{*} M_{L}\left(\hat{U}-M_{L}^{-1} \tilde{g}_{L}\right)-\left.\tilde{g}_{L}^{*} M_{L}^{-1} \tilde{g}_{L}\right|_{x_{L}} \\
& B T_{R}=-\left(\hat{U}-M_{R}^{-1} \tilde{g}_{R}\right)^{*} M_{R}\left(\hat{U}-M_{R}^{-1} \tilde{g}_{R}\right)+\left.\tilde{g}_{R}^{*} M_{R}^{-1} \tilde{g}_{R}\right|_{x_{R}} .
\end{aligned}
$$

\subsubsection{Choice of penalty parameters for the discrete formulation}

Let the penalty parameters be $\tau_{0}=\tilde{\tau}_{0} S_{L}, \tau_{N}=\tilde{\tau}_{N} S_{R}$ and $\sigma_{0, N}=0$. Inserting this into (30) and (31) yields

$$
\begin{aligned}
B T_{L}^{D} & =\left[\begin{array}{c}
\hat{V}_{0} \\
(\bar{D} \hat{V})_{0}
\end{array}\right]^{*}\left[\begin{array}{cc}
A+\tilde{\tau}_{0} \widetilde{H}_{L}+\left(\tilde{\tau}_{0} \widetilde{H}_{L}\right)^{*} & \left(\tilde{\tau}_{0}-I\right) B \\
\left(\tilde{\tau}_{0}^{*}-I\right) B & 0_{2}
\end{array}\right]\left[\begin{array}{c}
\hat{V}_{0} \\
(\bar{D} \hat{V})_{0}
\end{array}\right] \\
& -\hat{V}_{0}^{*} \tilde{\tau}_{0} \tilde{g}_{L}-\left(\hat{V}_{0}^{*} \tilde{\tau}_{0} \tilde{g}_{L}\right)^{*}
\end{aligned}
$$

and

$$
\begin{aligned}
B T_{R}^{D} & =\left[\begin{array}{c}
\hat{V}_{N} \\
(\bar{D} \hat{V})_{N}
\end{array}\right]^{*}\left[\begin{array}{cc}
-A+\tilde{\tau}_{N} \widetilde{H}_{R}+\left(\tilde{\tau}_{N} \widetilde{H}_{R}\right)^{*} & \left(\tilde{\tau}_{N}+I\right) B \\
\left(\tilde{\tau}_{N}^{*}+I\right) B & 0_{2}
\end{array}\right]\left[\begin{array}{c}
\hat{V}_{N} \\
(\bar{D} \hat{V})_{N}
\end{array}\right] \\
& -\hat{V}_{N}^{*} \tilde{\tau}_{N} \tilde{g}_{R}-\left(\hat{V}_{N}^{*} \tilde{\tau}_{N} \tilde{g}_{R}\right)^{*},
\end{aligned}
$$

respectively.

Proposition 6.3. Choosing the left penalty parameters as $\tau_{0}=S_{L}$ (i.e. $\tilde{\tau}_{0}=I$ ), where $S_{L}$ is given in (33), and $\sigma_{0}=0_{2}$, yields a stable numerical scheme, given that Proposition 6.1 holds and under the assumption that the right boundary terms are bounded as well.

Proof. Inserting $\tilde{\tau}_{0}=I$ into (37), the left discrete boundary term becomes

$$
B T_{L}^{D}=\hat{V}_{0}^{*}\left(A+\widetilde{H}_{L}+\widetilde{H}_{L}^{*}\right) \hat{V}_{0}-\hat{V}_{0}^{*} \tilde{g}_{L}-\tilde{g}_{L}^{*} \hat{V}_{0}
$$

and according to Proposition $6.1 M_{L}=A+\widetilde{H}_{L}+\widetilde{H}_{L}^{*}$ can be designed to be negative semi-definite. 
Remark: As previously discussed, the choice of $a$ and $b$ in $S_{L}$ is irrelevant for the continuous problem, while a valid choice is crucial for numerical stability.

Proposition 6.4. Choosing the right penalty parameters as $\tau_{N}=-S_{R}$ (i.e. $\tilde{\tau}_{N}=-I$ ), where $S_{R}$ is given in (33), and $\sigma_{N}=[0,0]^{T}$, yields a stable numerical scheme, under the assumption that the left boundary terms are bounded as well.

Proof. Inserting $\tilde{\tau}_{N}=-I$ into (38), the right discrete boundary term becomes

$$
B T_{R}^{D}=-\hat{V}_{N}^{*}\left(A+\widetilde{H}_{R}+\widetilde{H}_{R}^{*}\right) \hat{V}_{N}+\hat{V}_{N}^{*} \tilde{g}_{R}+\tilde{g}_{R}^{*} \hat{V}_{N}
$$

and according to Proposition $6.2 M_{R}=A+\widetilde{H}_{R}+\widetilde{H}_{R}^{*}$ is positive semi-definite.

Remark: Note that when using the penalty parameters as specified in Proposition 6.3 and 6.4, the discrete boundary terms (39) and (40) mimics the continuous ones perfectly, c.f. equation (34), and that stability of the numerical scheme is guaranteed.

\subsection{Replacing the ingoing variables}

\subsubsection{The continuous formulation}

Consider the matrix $\widetilde{A}$ in (17), and assume that we have found a rotation such that $\widetilde{A}=X \Lambda X^{T}$, where $\Lambda$ is diagonal. Note that the elements of $\Lambda$ are not necessarily the eigenvalues of $\widetilde{A}$, and that the vectors in $X$ may then not be orthogonal. According to Sylvester's law of inertia, the matrices $\widetilde{A}$ and $\Lambda$ will always have the same number of positive/negative eigenvalues for a non-singular $X$. The matrix $\Lambda$ has two positive entries and one negative entry for $v>0$, and is sorted as $\Lambda=\operatorname{diag}\left(\Lambda_{+}, \Lambda_{-}\right)$. The vectors are divided correspondingly, $X=\left[x_{+}, x_{-}\right]$, and the boundary terms in (17) are rewritten as

$$
\begin{aligned}
& B T_{L}=\left(X^{T} \bar{U}\right)^{*} \Lambda X^{T} \bar{U}=\left(x_{+}^{T} \bar{U}\right)^{*} \Lambda_{+} x_{+}^{T} \bar{U}+\left(x_{-}^{T} \bar{U}\right)^{*} \Lambda_{-} x_{-}^{T} \bar{U} \\
& B T_{R}=-\left(X^{T} \bar{U}\right)^{*} \Lambda X^{T} \bar{U}=-\left(x_{+}^{T} \bar{U}\right)^{*} \Lambda_{+} x_{+}^{T} \bar{U}-\left(x_{-}^{T} \bar{U}\right)^{*} \Lambda_{-} x_{-}^{T} \bar{U} .
\end{aligned}
$$

$x_{+}^{T} \bar{U}$ represents two right-going variables (ingoing at the left boundary), and $x_{-}^{T} \bar{U}$ represents one left-going variable (ingoing at the right boundary). The ingoing variables are given data in terms of known functions and outgoing variables, as

$$
x=x_{L}: \quad x_{+}^{T} \bar{U}+R_{L} x_{-}^{T} \bar{U}=\tilde{g}_{L}, \quad x=x_{R}: \quad x_{-}^{T} \bar{U}+R_{R} x_{+}^{T} \bar{U}=\tilde{g}_{R},
$$

where the matrices $R_{L, R}$ must be sufficiently small. Since we want to impose the nonreflecting boundary conditions $\bar{L}_{L} \bar{U}=\hat{g}_{L}$ and $\bar{L}_{R} \bar{U}=\hat{g}_{R}$, given in (3), we relate them to the general ones in (42) using scaling matrices. Denoting the scaling matrices $J_{L, R}$, we obtain

$$
\bar{L}_{L}=J_{L}\left(x_{+}^{T}+R_{L} x_{-}^{T}\right), \quad \bar{L}_{R}=J_{R}\left(x_{-}^{T}+R_{R} x_{+}^{T}\right),
$$

and identify $\tilde{g}_{L}=J_{L}^{-1} \hat{g}_{L}$ and $\tilde{g}_{R}=J_{R}^{-1} \hat{g}_{R}$. The matrices $J_{L, R}$ and $R_{L, R}$ can be computed from $x_{ \pm}$, which is assumed known, and $\bar{L}_{L, R}$, which are given in (20). Inserting the 
relations $x_{+}^{T} \bar{U}=\tilde{g}_{L}-R_{L} x_{-}^{T} \bar{U}$ and $x_{-}^{T} \bar{U}=\tilde{g}_{R}-R_{R} x_{+}^{T} \bar{U}$ from (42), into the boundary terms $B T_{L}$ and $B T_{R}$ in (41), respectively, yields

$$
\begin{aligned}
B T_{L} & =\left(x_{-}^{T} \bar{U}-\mathcal{C}_{L}^{-1} R_{L}^{*} \Lambda_{+} \tilde{g}_{L}\right)^{*} \mathcal{C}_{L}\left(x_{-}^{T} \bar{U}-\mathcal{C}_{L}^{-1} R_{L}^{*} \Lambda_{+} \tilde{g}_{L}\right) \\
& +\tilde{g}_{L}^{*}\left(\Lambda_{+}-\Lambda_{+} R_{L} \mathcal{C}_{L}^{-1} R_{L}^{*} \Lambda_{+}\right) \tilde{g}_{L} \\
B T_{R} & =-\left(x_{+}^{T} \bar{U}-\mathcal{C}_{R}^{-1} R_{R}^{*} \Lambda_{-} \tilde{g}_{R}\right)^{*} \mathcal{C}_{R}\left(x_{+}^{T} \bar{U}-\mathcal{C}_{R}^{-1} R_{R}^{*} \Lambda_{-} \tilde{g}_{R}\right) \\
& -\tilde{g}_{R}^{*}\left(\Lambda_{-}-\Lambda_{-} R_{R} \mathcal{C}_{R}^{-1} R_{R}^{*} \Lambda_{-}\right) \tilde{g}_{R}
\end{aligned}
$$

where $\mathcal{C}_{L}=R_{L}^{*} \Lambda_{+} R_{L}+\Lambda_{-}$and $\mathcal{C}_{R}=\Lambda_{+}+R_{R}^{*} \Lambda_{-} R_{R}$. For an energy estimate of the continuous problem $\mathcal{C}_{L} \leq 0$ and $\mathcal{C}_{R} \geq 0$ are necessary.

Proposition 6.5. The scalar $\mathcal{C}_{L}$ in (44) is non-positive, and hence the non-reflecting boundary condition (14) at the left boundary leads to an energy estimate.

Proof. Recall that $\widetilde{A}=x_{+} \Lambda_{+} x_{+}^{T}+x_{-} \Lambda_{-} x_{-}^{T}$. Starting from $\mathcal{A}_{L}$ in (18), we have

$$
\begin{aligned}
\mathcal{A}_{L} & =\Psi_{3}^{*} \widetilde{A} \Psi_{3} \\
& =\Psi_{3}^{*}\left(x_{-} \Lambda_{-} x_{-}^{T}+x_{-} R_{L}^{*} \Lambda_{+} R_{L} x_{-}^{T}\right) \Psi_{3}+\Psi_{3}^{*}\left(x_{+} \Lambda_{+} x_{+}^{T}-x_{-} R_{L}^{*} \Lambda_{+} R_{L} x_{-}^{T}\right) \Psi_{3} \\
& =\Psi_{3}^{*} x_{-} \mathcal{C}_{L} x_{-}^{T} \Psi_{3}+\underbrace{\Psi_{3}^{*}\left(x_{+} \Lambda_{+} x_{+}^{T}-x_{-} R_{L}^{*} \Lambda_{+} R_{L} x_{-}^{T}\right) \Psi_{3}}_{\mathcal{N}_{L}} .
\end{aligned}
$$

Inserting the relation $x_{+}^{T}+R_{L} x_{-}^{T}=J_{L}^{-1} \bar{L}_{L}$ from (43) into $\mathcal{N}_{L}$ we get

$$
\begin{aligned}
\mathcal{N}_{L} & =\Psi_{3}^{*}\left(x_{+} \Lambda_{+} x_{+}^{T}-x_{-} R_{L}^{*} \Lambda_{+} R_{L} x_{-}^{T}\right) \Psi_{3} \\
& =\frac{1}{2} \Psi_{3}^{*}\left(\left(x_{+}^{T}+R_{L} x_{-}^{T}\right)^{*} \Lambda_{+}\left(x_{+}^{T}-R_{L} x_{-}^{T}\right)+\left(x_{+}^{T}-R_{L} x_{-}^{T}\right)^{*} \Lambda_{+}\left(x_{+}^{T}+R_{L} x_{-}^{T}\right)\right) \Psi_{3} \\
\text { use }(43)] & =\frac{1}{2} \Psi_{3}^{*}\left(\left(J_{L}^{-1} \bar{L}_{L}\right)^{*} \Lambda_{+}\left(x_{+}^{T}-R_{L} x_{-}^{T}\right)+\left(x_{+}^{T}-R_{L} x_{-}^{T}\right)^{*} \Lambda_{+} J_{L}^{-1} \bar{L}_{L}\right) \Psi_{3} \\
& =\left(\bar{L}_{L} \Psi_{3}\right)^{*} \frac{\left(J_{L}^{-1}\right)^{*} \Lambda_{+}\left(x_{+}^{T}-R_{L} x_{-}^{T}\right) \Psi_{3}}{2}+\frac{\Psi_{3}^{*}\left(x_{+}^{T}-R_{L} x_{-}^{T}\right)^{*} \Lambda_{+} J_{L}^{-1}}{2} \bar{L}_{L} \Psi_{3} .
\end{aligned}
$$

Recall that $\bar{L}_{L}=\left[\Phi_{1}, \Phi_{2}\right]^{T}$ and $\Phi_{j}^{T} \Psi_{i}=0$ such that $\bar{L}_{L} \Psi_{3}=0$. This leads to $\mathcal{N}_{L}=0$ and finally the relation $\mathcal{A}_{L}=\left(x_{-}^{T} \Psi_{3}\right)^{*} \mathcal{C}_{L} x_{-}^{T} \Psi_{3}$, where $x_{-}^{T} \Psi_{3} \neq 0$. Since $\mathcal{A}_{L} \leq 0$ from Proposition 4.2 , we know that $\mathcal{C}_{L} \leq 0$ as well.

Proposition 6.6. The matrix $\mathcal{C}_{R}$ in (44) is non-negative, and hence the non-reflecting boundary condition (14) at the right boundary leads to an energy estimate.

The proof of Proposition 6.6 is similar to that of Proposition 6.5, and is given in Appendix C.3.

\subsubsection{Choice of penalty parameters for the discrete formulation}

The continuous boundary terms in (41) depend on $\bar{U}=\left[\hat{p}, \hat{u}, \hat{u}_{x}\right]^{T}$, while the discrete boundary terms in (30) and (31) depend on $\hat{V}_{j}=\left[\hat{p}_{j}, \hat{u}_{j}\right]^{T}$ and $(\bar{D} \hat{V})_{j}=\left[(D \hat{p})_{j},(D \hat{u})_{j}\right]^{T}$, 
( $j$ being 0 or $N)$. The additional dependence on $(D \hat{p})_{0}$ and $(D \hat{p})_{N}$ will be removed. The penalty parameters are

$$
\tau_{0}=\left[\begin{array}{cc}
\tau_{0}^{11} & \tau_{0}^{12} \\
\tau_{0}^{21} & \tau_{0}^{22}
\end{array}\right], \quad \sigma_{0}=\left[\begin{array}{cc}
\sigma_{0}^{11} & \sigma_{0}^{12} \\
\sigma_{0}^{21} & \sigma_{0}^{22}
\end{array}\right], \quad \tau_{N}=\left[\begin{array}{c}
\tau_{N}^{11} \\
\tau_{N}^{21}
\end{array}\right], \quad \sigma_{N}=\left[\begin{array}{c}
\sigma_{N}^{11} \\
\sigma_{N}^{21}
\end{array}\right] .
$$

Zeroing out the first row of $\sigma_{0, N}$ such that $\sigma_{0}^{11}=\sigma_{0}^{12}=0$ and $\sigma_{N}^{11}=0$, the boundary terms in (30) and (31) become independent on $(D \hat{p})_{0}$ and $(D \hat{p})_{N}$, respectively. Denoting the remaining rows $\tilde{\sigma}_{0}=\left[\sigma_{0}^{21}, \sigma_{0}^{22}\right]$ and $\tilde{\sigma}_{N}=\left[\sigma_{N}^{21}\right]$, the boundary terms in (30) and (31) can be written

$$
\begin{aligned}
B T_{L}^{D} & =\bar{V}_{0}^{*}\left[\widetilde{A}+\left[\begin{array}{l}
\tau_{0} \\
\tilde{\sigma}_{0}
\end{array}\right] \bar{L}_{L}+\left(\left[\begin{array}{l}
\tau_{0} \\
\tilde{\sigma}_{0}
\end{array}\right] \bar{L}_{L}\right)^{*}\right] \bar{V}_{0} \\
& -\bar{V}_{0}^{*}\left[\begin{array}{c}
\tau_{0} \\
\tilde{\sigma}_{0}
\end{array}\right] \hat{g}_{L}-\left(\bar{V}_{0}^{*}\left[\begin{array}{c}
\tau_{0} \\
\tilde{\sigma}_{0}
\end{array}\right] \hat{g}_{L}\right)^{*}
\end{aligned}
$$

and

$$
\begin{aligned}
B T_{R}^{D} & =\bar{V}_{N}^{*}\left[-\widetilde{A}+\left[\begin{array}{c}
\tau_{N} \\
\tilde{\sigma}_{N}
\end{array}\right] \bar{L}_{R}+\left(\left[\begin{array}{c}
\tau_{N} \\
\tilde{\sigma}_{N}
\end{array}\right] \bar{L}_{R}\right)^{*}\right] \bar{V}_{N} \\
& -\bar{V}_{N}^{*}\left[\begin{array}{c}
\tau_{N} \\
\tilde{\sigma}_{N}
\end{array}\right] \hat{g}_{R}-\left(\bar{V}_{N}^{*}\left[\begin{array}{c}
\tau_{N} \\
\tilde{\sigma}_{N}
\end{array}\right] \hat{g}_{R}\right)^{*},
\end{aligned}
$$

respectively, where $\bar{V}_{0}=\left[\hat{p}_{0}, \hat{u}_{0},(D \hat{u})_{0}\right]^{T}$ and $\bar{V}_{N}=\left[\hat{p}_{N}, \hat{u}_{N},(D \hat{u})_{N}\right]^{T}$.

Proposition 6.7. Choosing the penalty parameter elements $\tau_{0}^{i j}$ and $\sigma_{0}^{i j}$ in (45) as

$$
\sigma_{0}^{11}=\sigma_{0}^{12}=0, \quad\left[\begin{array}{cc}
\tau_{0}^{11} & \tau_{0}^{12} \\
\tau_{0}^{21} & \tau_{0}^{22} \\
\sigma_{0}^{21} & \sigma_{0}^{22}
\end{array}\right]=-x_{+} \Lambda_{+} J_{L}^{-1}
$$

results in a strongly stable numerical scheme.

Proof. Inserting the specific choice $\left[\tau_{0}^{T}, \tilde{\sigma}_{0}^{T}\right]^{T}=-x_{+} \Lambda_{+} \bar{J}_{L}^{-1}$ into (46) yields

$$
\begin{aligned}
B T_{L}^{D} & =\left(x_{-}^{T} \bar{V}_{0}-\mathcal{C}_{L}^{-1} R_{L}^{*} \Lambda_{+} \tilde{g}_{L}\right)^{*} \mathcal{C}_{L}\left(x_{-}^{T} \bar{V}_{0}-\mathcal{C}_{L}^{-1} R_{L}^{*} \Lambda_{+} \tilde{g}_{L}\right) \\
& +\tilde{g}_{L}^{*}\left(\Lambda_{+}-\Lambda_{+} R_{L} \mathcal{C}_{L}^{-1} R_{L}^{*} \Lambda_{+}\right) \tilde{g}_{L} \\
& -\left(\bar{L}_{L} \bar{V}_{0}-\hat{g}_{L}\right)^{*} J_{L}^{-*} \Lambda_{+} J_{L}^{-1}\left(\bar{L}_{L} \bar{V}_{0}-\hat{g}_{L}\right)
\end{aligned}
$$

where, according to Proposition $6.5, \mathcal{C}_{L} \leq 0$.

Proposition 6.8. Choosing the penalty parameter elements $\tau_{N}^{i j}$ and $\sigma_{N}^{i j}$ in (45) as

$$
\sigma_{N}^{11}=0, \quad\left[\begin{array}{c}
\tau_{N}^{11} \\
\tau_{N}^{21} \\
\sigma_{N}^{21}
\end{array}\right]=x_{-} \Lambda_{-} J_{R}^{-1}
$$

results in a strongly stable numerical scheme. 
Proof. Inserting the penalty parameters $\left[\tau_{N}^{T}, \tilde{\sigma}_{N}^{T}\right]^{T}=x_{-} \Lambda_{-} \bar{J}_{R}^{-1}$ into (47), yields

$$
\begin{aligned}
B T_{R}^{D} & =-\left(x_{+}^{T} \bar{V}_{N}-\mathcal{C}_{R}^{-1} R_{R}^{*} \Lambda_{-} \tilde{g}_{R}\right)^{*} \mathcal{C}_{R}\left(x_{+}^{T} \bar{V}_{N}-\mathcal{C}_{R}^{-1} R_{R}^{*} \Lambda_{-} \tilde{g}_{R}\right) \\
& -\tilde{g}_{R}^{*}\left(\Lambda_{-}-\Lambda_{-} R_{R} \mathcal{C}_{R}^{-1} R_{R}^{*} \Lambda_{-}\right) \tilde{g}_{R} \\
& +\left(\bar{L}_{R} \bar{V}_{N}-\hat{g}_{R}\right)^{*} J_{R}^{-*} \Lambda_{-} J_{R}^{-1}\left(\bar{L}_{R} \bar{V}_{N}-\hat{g}_{R}\right)
\end{aligned}
$$

where $\mathcal{C}_{R} \geq 0$ according to Proposition 6.6.

Remark: Note that when using the penalty parameters as specified in Proposition 6.7 and 6.8, the discrete boundary terms $B T_{L, R}^{D}$ in (48) and (49) correspond exactly to the continuous boundary terms $B T_{L, R}$ in (44), except for a small damping term. The damping term is a function of the deviation from the boundary data, and goes to zero as the mesh is refined.

\section{Implementation details}

Here we describe the numerical procedure, including how the Laplace transform is inverted. As an example, we consider imposing the Dirichlet boundary conditions at the left boundary, and using the exact NRBC at the right boundary. Hence the term $\left(\Sigma_{0} * V\right)(t)=\mathcal{L}^{-1}\left\{\hat{\Sigma}_{0}(s) \hat{V}(s)\right\}$ in $(23)$ will be replaced by

$$
\left(P^{-1} e_{0} \otimes \tau_{0}^{\text {Dir. }}+P^{-1} D^{T} e_{0} \otimes \sigma_{0}^{\text {Dir. }}\right)\left(L_{L} V_{0}-g_{L}\right) .
$$

Giving Dirichlet boundary conditions such that $p=g_{1}$ and $u=g_{2}$ at the left boundary, implies that $L_{L}=I_{2}$. The penalty matrices in (50) are chosen as specified in Appendix D.1.

\subsection{Inverting the Laplace transform}

At the right boundary we impose the non-reflecting boundary conditions. The convolution $\left(\Sigma_{N} * V\right)(t)=\mathcal{L}^{-1}\left\{\hat{\Sigma}_{N}(s) \hat{V}(s)\right\}$ in (23) is defined as

$$
\mathcal{L}^{-1}\left\{\hat{\Sigma}_{N}(s) \hat{V}(s)\right\}=\int_{0}^{t} \Sigma_{N}(\tau) V(t-\tau) d \tau \text {. }
$$

We follow the work in $[20,21]$, and approximate the integral (51) at time $t_{n}=n h$ by the convolution quadrature

$$
\sum_{j=0}^{n} \omega_{j}(h) V\left(t_{n-j}\right)
$$

where $h$ is the time step, and where $\omega_{j}(h) \approx h \Sigma_{N}\left(t_{j}\right)$ for $j h$ away from zero. The coefficients $\omega_{j}(h)$ in (52) are approximated by

$$
\hat{\omega}_{j}(h)=\rho^{-j} \frac{1}{L} \sum_{l=0}^{L-1} \hat{\Sigma}_{N}\left(\frac{\delta\left(\rho e^{i \tau_{l}}\right)}{h}\right) e^{-i j \tau_{l}}, \quad \tau_{l}=2 \pi l / L .
$$

The constants $\rho$ and $L$ and the function $\delta$ must be suitably chosen. We use $\rho=0.975$, $L=T / h$, where $T$ is the end time of the computation, and $\delta(\zeta)=\sum_{i=1}^{3} \frac{1}{i}(1-\zeta)^{i}$.

Remark: Note that there exist more elaborate versions of this method, see e.g. [22]. 


\subsection{Time discretization}

We let the boundary data $\hat{g}_{R}$ be zero such that $\hat{\Gamma}_{N}=0$ in $(25)$ and $\Gamma_{N}=0$ in $(23)$. The semi-discrete scheme (23) is then expressed as

$$
V_{t}=\mathbf{F}(t, V) \text {, }
$$

such that

$$
\mathbf{F}(t, V)=\mathbf{A} V+\mathbf{G}(t)+\int_{0}^{t} \Sigma_{N}(\tau) V(t-\tau) d \tau
$$

where, including the Dirichlet boundary condition in (50),

$$
\begin{aligned}
\mathbf{A} & =-(D \otimes A)+\left(D^{2} \otimes B\right)+\left(P^{-1} e_{0} \otimes \tau_{0}^{\text {Dir. }}+P^{-1} D^{T} e_{0} \otimes \sigma_{0}^{D i r .}\right)\left(e_{0}^{T} \otimes L_{L}\right) \\
\mathbf{G}(t) & =F-\left(P^{-1} e_{0} \otimes \tau_{0}^{D i r .}+P^{-1} D^{T} e_{0} \otimes \sigma_{0}^{\text {Dir. }}\right) g_{L}(t) .
\end{aligned}
$$

The ordinary differential equation (54) is discretized in time using the trapezoidal rule,

$$
V_{n+1}=V_{n}+\frac{h}{2}\left(\mathbf{F}\left(t_{n}, V_{n}\right)+\mathbf{F}\left(t_{n+1}, V_{n+1}\right)\right) .
$$

We insert (55) into (56), and use the approximation

$$
\int_{0}^{t} \Sigma_{N}(\tau) V(t-\tau) d \tau \approx \sum_{j=0}^{n} \hat{\omega}_{j}(h) V\left(t_{n-j}\right) .
$$

After moving all terms containing $V_{n+1}$ to the left-hand side, we obtain the final scheme

$$
\begin{aligned}
\left(I-\frac{h}{2}\left(\mathbf{A}+\hat{\omega}_{0}\right)\right) V_{n+1} & =\left(I+\frac{h}{2} \mathbf{A}\right) V_{n}+\frac{h}{2} \sum_{j=0}^{n}\left(\hat{\omega}_{j}+\hat{\omega}_{j+1}\right) V_{n-j} \\
& +\frac{h}{2}\left(\mathbf{G}\left(t_{n}\right)+\mathbf{G}\left(t_{n+1}\right)\right) .
\end{aligned}
$$

When computing $\hat{\omega}_{j}$ in (57), using (53), we need $\hat{\Sigma}_{N}$. We rewrite the parts of $\hat{\Sigma}_{N} \hat{V}$ in (28) such that we can identify

$$
\hat{\Sigma}_{N}=\bar{P}^{-1}\left(E_{N} \otimes \tau_{N} H_{R}+D^{T} E_{N} \otimes \sigma_{N} H_{R}+E_{N} D \otimes \tau_{N} G_{R}+D^{T} E_{N} D \otimes \sigma_{N} G_{R}\right),
$$

where $E_{N}=e_{N} e_{N}^{T}$. That is, $\hat{\Sigma}_{N}$ is a $2(N+1) \times 2(N+1)$ matrix, and consequently so are $\hat{\omega}_{j}$. Fortunately $\hat{\Sigma}_{N}$ is sparse since $E_{N}$ mainly consist of zeroes, and it suffice to compute the lower right corner of $\hat{\omega}_{j}$.

Remark: The scheme (57) exemplifies the special case when having the Dirichlet boundary conditions at the left boundary and the exact NRBC at the right boundary. Other scenarios, for example when having the exact NRBC's at the left boundary and the Dirichlet boundary condition at the right boundary, are derived in a similar way. 


\subsection{Computation of the penalty parameters for the NRBC's}

When computing $\hat{\Sigma}_{N}$ in (58), we need the penalty parameters $\tau_{N}$ and $\sigma_{N}$, which will be given explicitly below. In the numerical simulations we will also impose the exact NRBC's at the left boundary, using $\hat{\Sigma}_{0}$ (i.e. the left analogue to $\hat{\Sigma}_{N}$ ), and therefore we will present $\tau_{0}$ and $\sigma_{0}$ as well. We either replace the indefinite terms, using

$$
\tau_{0}=S_{L}, \quad \sigma_{0}=\left[\begin{array}{ll}
0 & 0 \\
0 & 0
\end{array}\right], \quad \tau_{N}=-\left[\begin{array}{l}
0 \\
1
\end{array}\right], \quad \sigma_{N}=\left[\begin{array}{l}
0 \\
0
\end{array}\right],
$$

(see Propositions 6.3 and 6.4), or replace the ingoing variables, using

$$
\begin{array}{ll}
\tau_{0}=-\left[\begin{array}{ll}
v+c & 0 \\
v+c & 0
\end{array}\right] \Lambda_{+} J_{L}^{-1}, & \sigma_{0}=-\left[\begin{array}{cc}
0 & 0 \\
-\varepsilon & \varepsilon
\end{array}\right] \Lambda_{+} J_{L}^{-1} \\
\tau_{N}=\left[\begin{array}{l}
v-c \\
c-v
\end{array}\right] \Lambda_{-} J_{R}^{-1}, & \sigma_{N}=\left[\begin{array}{l}
0 \\
\varepsilon
\end{array}\right] \Lambda_{-} J_{R}^{-1} .
\end{array}
$$

The derivation of the penalty parameters (60) is described below. We first find $J_{L, R}, R_{L, R}$ by rewriting (43) as

$$
\bar{L}_{L}=\left[\begin{array}{lll}
J_{L} & J_{L} R_{L}
\end{array}\right] X^{T}, \quad \bar{L}_{R}=\left[J_{R} R_{R} J_{R}\right] X^{T} .
$$

The rotation $\widetilde{A}=X \Lambda X^{T}$, where $\Lambda=\operatorname{diag}\left(\Lambda_{+}, \Lambda_{-}\right)$and $X=\left[x_{+}, x_{-}\right]$, can be chosen in numerous ways, and the choice of rotation will influence the penalty parameters slightly. (To compute the eigenvalues and eigenvectors numerically is one option.) We have used the rotation

$$
\Lambda_{+}=\left[\begin{array}{cc}
\frac{1}{2(v+c)} & \\
& \frac{-v}{v^{2}-c^{2}}
\end{array}\right], \quad \Lambda_{-}=\left[\frac{1}{2(v-c)}\right], \quad x_{+}=\left[\begin{array}{cc}
v+c & 0 \\
v+c & 0 \\
-\varepsilon & \varepsilon
\end{array}\right], \quad x_{-}=\left[\begin{array}{c}
v-c \\
c-v \\
\varepsilon
\end{array}\right],
$$

which is valid for $0<v<c$ and yields

$$
X^{-T}=\left[\begin{array}{ccc}
\frac{1}{2(v+c)} & \frac{-c}{v^{2}-c^{2}} & \frac{1}{2(v-c)} \\
\frac{1}{2(v+c)} & \frac{v}{v^{2}-c^{2}} & \frac{-1}{2(v-c)} \\
0 & \frac{1}{\varepsilon} & 0
\end{array}\right]
$$

The matrices $J_{L, R}$ are obtained from $\bar{L}_{L, R} X^{-T}$, where $\bar{L}_{L, R}$ are given in (20), as

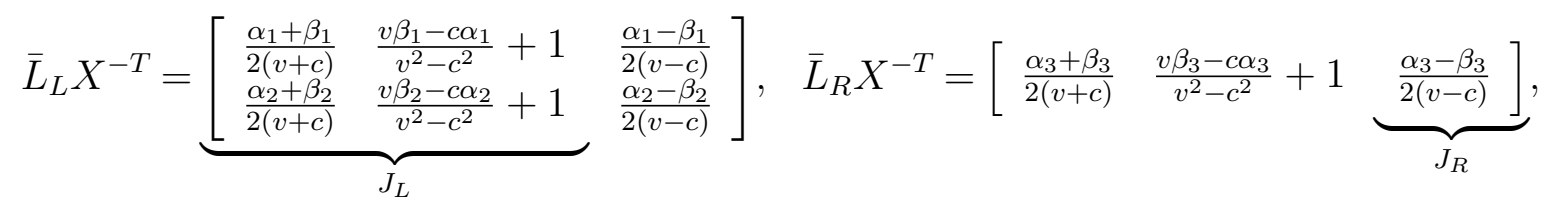

and thereafter the penalty parameters are computed as specified in Proposition 6.7 and Proposition 6.8, such that (60) is obtained.

Remark: In the numerical experiments we will compare the exact NRBC's to a low order approximation of the NRBC's. The approximative NRBC's are derived by inserting $s=0$ into the exact non-reflecting boundary operators, which yields time-local, low-reflecting boundary conditions. The penalty parameters are obtained similarly, by inserting $s=0$ into $\tau_{0, N}$ and $\sigma_{0, N}$. The resulting operators are given in Appendix D.2. 


\section{Numerical results}

We let the computational domain be $\left[x_{L}, x_{R}\right]=[0,1]$, and as reference solution we use the solution from a five times larger domain. The errors are defined as the difference between the solution and the reference solution, as $\Delta p=p-p^{\text {ref }}$ and $\Delta u=u-u^{\text {ref }}$. The SBP matrix $P$ is used for computing norms of the errors, as

$$
\operatorname{Error}(p)=\|\Delta p\|_{P}, \quad \operatorname{Error}(u)=\|\Delta u\|_{P},
$$

where the norm of a vector $\mathbf{v}$ is defined as $\|\mathbf{v}\|_{P}^{2}=\mathbf{v}^{T} P \mathbf{v}$. See [19] for details on the accuracy and interpretations of SBP norms. For the space discretization we use a third order accurate SBP scheme, and as mentioned earlier, the trapezoidal rule is used for the time discretization. In all simulations we use the physical parameter values $c=1$, $v=0.5$ and $\varepsilon=0.1$. The time step is $h=0.001$ and the end time $T=0.4$. The number of grid point varies, but in the figures we have used $N=64$. The time step is sufficiently small, such that the errors from the space discretization are dominating. To reduce the number of figures we only show the solution for the variable $u$, but the results for the variable $p$ are similar and presented in the tables.

\subsection{Non-reflecting boundary conditions at the right boundary}

First, simulations are performed using the scheme (57) with the penalty parameters given in (59) and (60). As initial condition we use

$$
p(x, 0)=u(x, 0)=\left\{\begin{array}{cr}
0 & 0.05 \geq x \\
\cos ^{3}(2.5 \pi(x-0.25)) & 0.05<x<0.45 \\
0 & x \geq 0.45 .
\end{array}\right.
$$

At the left boundary the Dirichlet boundary conditions are imposed and at the right boundary the solution is supposed to propagate out without reflections. This is the same problem setup as in the introducing examples in Figure 1 and Table 1. In comparison the exact NRBC outperforms those examples by far, see Figure 2 .

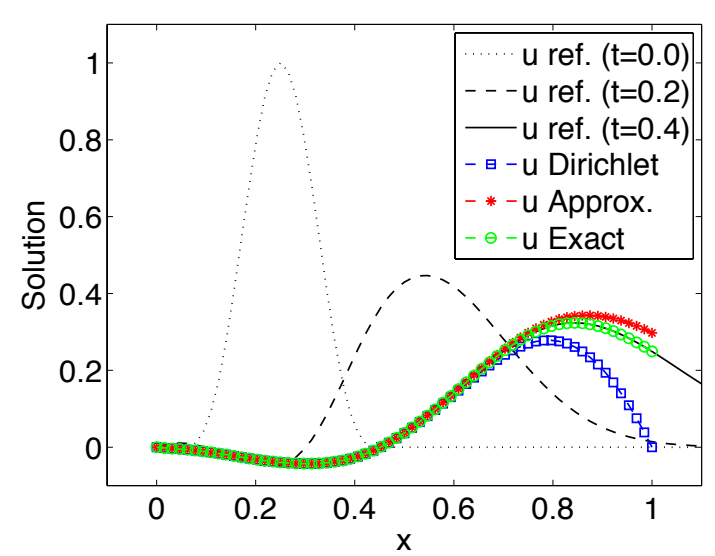

(a) $t=0.4$

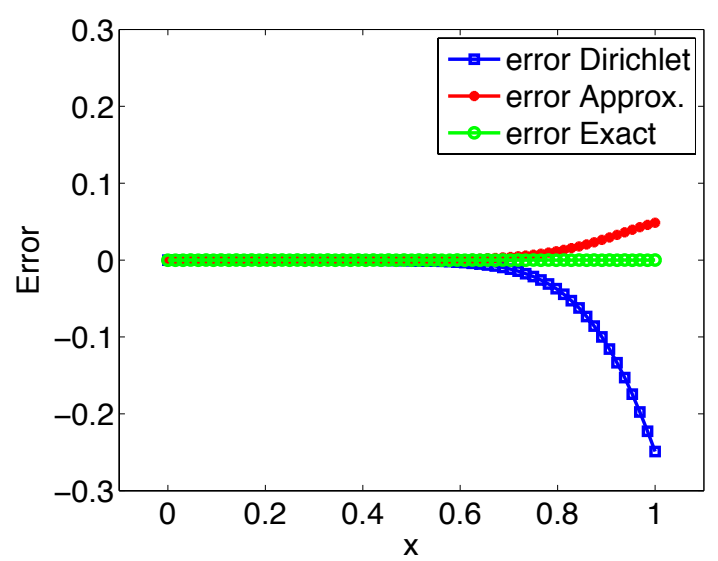

(b) $t=0.4$

Figure 2: The solution to (1) with initial condition given by (61). At $x=1$ the pulse should pass without reflections. At the right boundary the Dirichlet boundary condition, the approximate NRBC or the exact NRBC is used. 
More importantly, the exact NRBC solution converges to the reference solution as the mesh is refined, see Table 2 and 3. The errors have the same size, independently of whether the "replacing the indefinite terms" or the "replacing the ingoing variables" penalty is used. In the simulations, the computational cost when using the exact NRBC's are the same as when using any of the other boundary conditions.

\begin{tabular}{|c|ccc|ccc|}
\hline$N$ & Error $(p)$ & ratio & conv. rate & Error $(u)$ & ratio & conv. rate \\
\hline 16 & 0.00094582 & & & 0.00120231 & & \\
32 & 0.00010451 & 9.0497 & 3.1779 & 0.00014386 & 8.3575 & 3.0631 \\
64 & 0.00001193 & 8.7569 & 3.1304 & 0.00001848 & 7.7862 & 2.9609 \\
128 & 0.00000142 & 8.3796 & 3.0669 & 0.00000239 & 7.7320 & 2.9508 \\
\hline
\end{tabular}

Table 2: Results obtained using the exact NRBC (with the "replacing the indefinite terms" penalty) at the right boundary.

\begin{tabular}{|c|ccc|ccc|}
\hline$N$ & $\operatorname{Error}(p)$ & ratio & conv. rate & $\operatorname{Error}(u)$ & ratio & conv. rate \\
\hline 16 & 0.00091109 & & & 0.00121302 & & \\
32 & 0.00010158 & 8.9690 & 3.1649 & 0.00014664 & 8.2722 & 3.0483 \\
64 & 0.00001152 & 8.8217 & 3.1411 & 0.00001872 & 7.8317 & 2.9693 \\
128 & 0.00000139 & 8.2978 & 3.0527 & 0.00000241 & 7.7753 & 2.9589 \\
\hline
\end{tabular}

Table 3: Results obtained using the exact NRBC (with the "replacing the ingoing variables" penalty) at the right boundary.

\subsection{Non-reflecting boundary conditions at the left boundary}

Next we consider the NRBC's at the left boundary. For this case we use the initial condition

$$
p(x, 0)=-u(x, 0)=\left\{\begin{array}{cr}
0 & 0.3 \geq x \\
-\cos ^{3}(2.5 \pi(x-0.5)) & 0.3<x<0.7 \\
0 & x \geq 0.7,
\end{array}\right.
$$

such that the main content of the initial solution travels in the left direction. The resulting solution at time $t=0.4$ is shown in Figure 3, and as can be seen in Table 4 the solution converges to the reference solution as the mesh is refined.

\begin{tabular}{|c|ccc|ccc|}
\hline$N$ & $\operatorname{Error}(p)$ & ratio & conv. rate & $\operatorname{Error}(u)$ & ratio & conv. rate \\
\hline 16 & 0.00026816 & & & 0.00036867 & & \\
32 & 0.00003824 & 7.0134 & 2.8101 & 0.00005167 & 7.1355 & 2.8350 \\
64 & 0.00000414 & 9.2323 & 3.2067 & 0.00000522 & 9.9028 & 3.3078 \\
128 & 0.00000051 & 8.0689 & 3.0124 & 0.00000064 & 8.1321 & 3.0236 \\
\hline
\end{tabular}

Table 4: Results obtained using the exact NRBC (with the "replacing the ingoing variables" penalty) at the left boundary.

The results obtained using the "replacing the indefinite terms" are omitted since those are similar to the results obtained using the "replacing the ingoing terms" penalty. 


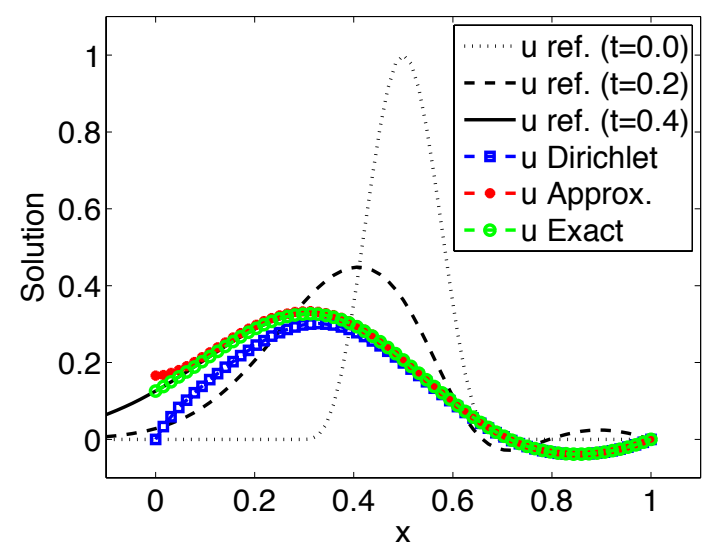

(a) $t=0.4$

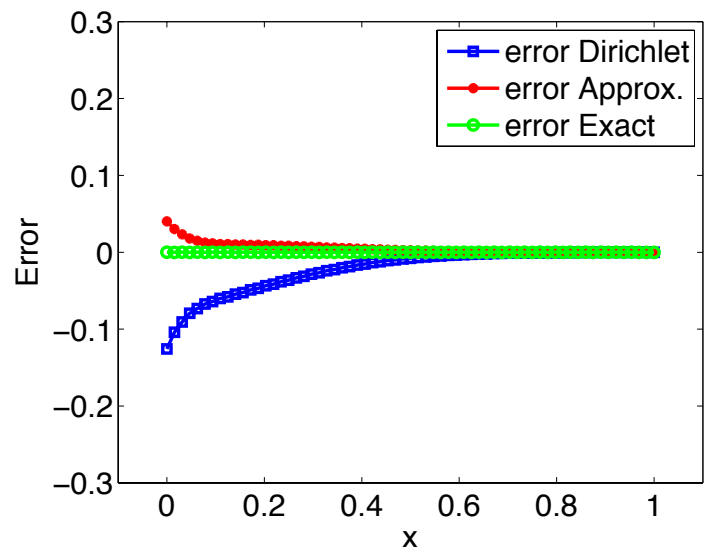

(b) $t=0.4$

Figure 3: The solution to (1) with initial condition given by (62). At $x=0$ the pulse should pass without reflections. At the left boundary the Dirichlet boundary condition, the approximate NRBC or the exact NRBC is imposed.

\subsection{Initial condition without compact support}

In the boundary conditions (14) the possibility of perturbed data, due to an unknown particular solution, is indicated. To model this, we also use an initial condition that does not have compact support in $x \in[0,1]$,

$$
p(x, 0)=u(x, 0)=\left\{\begin{array}{cr}
0 & 0.7 \geq x \\
\cos ^{3}(2.5 \pi(x-0.9)) & 0.7<x<1.1 \\
0 & x \geq 1.1
\end{array}\right.
$$

where $p(1,0)=u(1,0) \approx 0.35$. Despite this, we still give zero boundary data to the non-reflecting boundary condition (which we know is wrong, i.e. $g_{R}^{\prime}$ in (14) will be non-zero). The results for the exact NRBC's are still superior compared to the ones obtained with the Dirichlet or the approximate NRBC's, see Figure 4. However, since the boundary data does not match the non-zero particular solution, the convergence rates are zero.

\section{Conclusions}

We have investigated exact non-reflecting boundary conditions (NRBC) for flow problems, with focus on the theoretical aspects, well-posedness and stability. We consider an incompletely parabolic system of partial differential equations, as a model of the NavierStokes equations. The exact NRBC's were derived in Laplace transformed space.

We express the transformed solution as a superposition of ingoing and outgoing waves, and eliminate the ingoing waves at each boundary. Both inflow and outflow NRBC's are derived. It is shown that the exact non-reflecting boundary conditions lead to well-posedness, both in the GKS sense and in the energy sense.

The system is discretized in space using a high order accurate finite difference scheme on Summation-By-Parts form (SBP), and the boundary conditions are imposed 


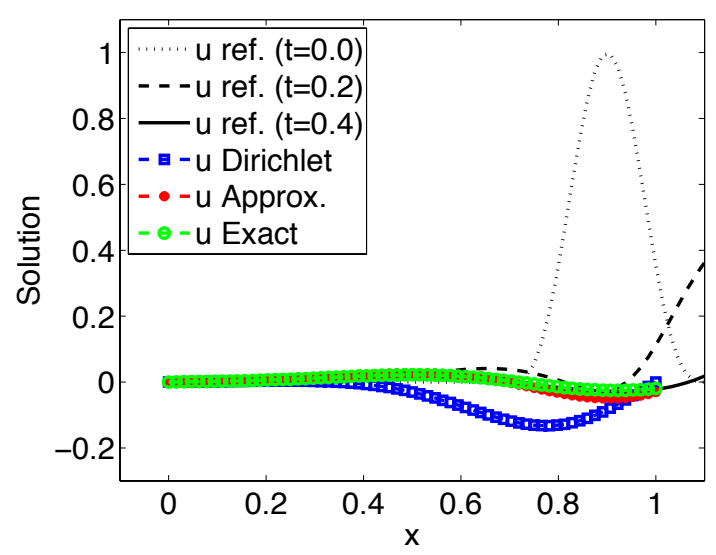

(a) $t=0.4$

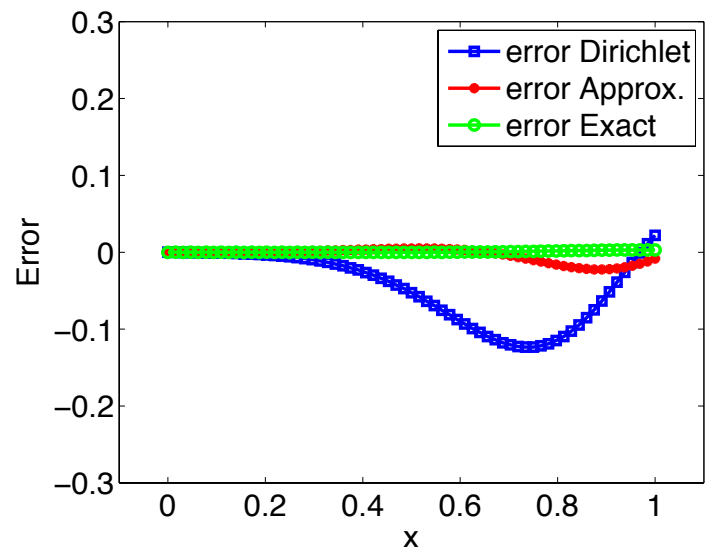

(b) $t=0.4$

Figure 4: The solution to (1) with initial condition given by (63). At $x=1$ the pulse should pass out without reflections. At the right boundary the Dirichlet boundary condition, the approximate NRBC or the exact NRBC is imposed.

weakly using a penalty formulation (SAT). With the continuous energy estimate as a guideline, two different SAT formulations have been derived, both yielding a discrete energy estimate mimicking the continuous one. Hence, by the combined use of the SBP operators and the SAT implementation, stability follows directly from the result of well-posedness for the continuous problem.

The exact non-reflecting boundary conditions are global in time, and must be transformed back for the numerical experiments. This is done by employing convolution quadratures. In the simulations the solutions converge to a reference solution, as accurately as the design order of the numerical scheme. The two different SAT formulations derived perform equally good, producing almost identical results in the numerical simulations.

We have compared the exact NRBC's to the Dirichlet boundary conditions and to approximate NRBC's. The exact NRBC's outperform the other conditions, yielding lower reflections both for exact and erroneous boundary data. Unlike the approximative non-reflecting boundary conditions and the Dirichlet boundary conditions, the exact ones yields convergence to the correct solution when the mesh is refined (and exact boundary data is available).

The superior accuracy, both on the boundary and in the interior (owing to the exact NRBC's and the high order scheme, respectively), in combination with the guaranteed stability, results in a competitive numerical method for computations on unbounded domains.

\section{A Multiple roots}

We show that the polynomial $q(\kappa, s)$ in (6) has no multiple roots $\kappa$ for $\operatorname{Re}(s) \geq 0$, unless $v=c$. We start by writing $\tilde{q}(\kappa, s)=-q(\kappa, s) /(\varepsilon v)$ as

$$
\tilde{q}(\kappa, s)=\kappa^{3}-r_{2} \kappa^{2}+r_{1} \kappa-r_{0}
$$


where the coefficients $r_{0}, r_{1}$ and $r_{2}$ are given in (9). The derivative $\tilde{q}^{\prime}(\kappa, s)=\frac{\partial}{\partial \kappa} \tilde{q}(\kappa, s)$,

$$
\tilde{q}^{\prime}(\kappa, s)=3 \kappa^{2}-2 r_{2} \kappa+r_{1},
$$

has roots

$$
\kappa_{4,5}=\frac{r_{2}}{3} \pm \sqrt{\left(\frac{r_{2}}{3}\right)^{2}-\frac{r_{1}}{3}} .
$$

If the polynomial $\tilde{q}(\kappa, s)$ has a multiple root $\kappa_{j}$, then that root $\kappa_{j}$ will be a solution to the derivative $\tilde{q}^{\prime}(\kappa, s)$ as well. To check whether $\tilde{q}(\kappa, s)$ and $\tilde{q}^{\prime}(\kappa, s)$ have any roots in common, we insert $\kappa_{4,5}$ into $\tilde{q}(\kappa, s)$. This yields

$$
\tilde{q}\left(\kappa_{4,5}, s\right)=\frac{-1}{27}\left(r_{2}\left(2 r_{2}^{2}-9 r_{1}\right) \pm 2 \sqrt{r_{2}^{2}-3 r_{1}}\left(r_{2}^{2}-3 r_{1}\right)+27 r_{0}\right) \text {. }
$$

Requiring $\tilde{q}\left(\kappa_{4,5}, s\right)=0$ leads to

$$
r_{2}\left(2 r_{2}^{2}-9 r_{1}\right)+27 r_{0}=\mp 2 \sqrt{r_{2}^{2}-3 r_{1}}\left(r_{2}^{2}-3 r_{1}\right)
$$

which we square on both sides to obtain

$$
\left(r_{2}\left(2 r_{2}^{2}-9 r_{1}\right)+27 r_{0}\right)^{2}=4\left(r_{2}^{2}-3 r_{1}\right)^{3} .
$$

If the relation (64) is fulfilled $q(\kappa, s)$ has a multiple root. We check if this can occur by defining $\Upsilon=\left(r_{2}\left(2 r_{2}^{2}-9 r_{1}\right)+27 r_{0}\right)^{2}-4\left(r_{2}^{2}-3 r_{1}\right)^{3}$, and see whether it is possible to find $\Upsilon=0$. Inserting the values $r_{0}=s^{2} /(\varepsilon v), r_{1}=-2 s / \varepsilon$ and $r_{2}=\left(v^{2}-c^{2}-s \varepsilon\right) /(\varepsilon v)$ from (9) gives

$$
\Upsilon=-27 \frac{s^{2}}{\varepsilon^{4} v^{4}}\left(4 c^{2}\left(v^{2}-c^{2}\right)^{2}+4 c^{2}\left(3 c^{2}+5 v^{2}\right) s \varepsilon+\left(v^{2}+12 c^{2}\right)(s \varepsilon)^{2}+4(s \varepsilon)^{3}\right) .
$$

Let $s \varepsilon=\tilde{\eta}+\tilde{\xi} i$ to split $\Upsilon$ into one real and one imaginary part, as

$$
\begin{aligned}
\Upsilon= & -27 \frac{s^{2}}{\varepsilon^{4} v^{4}}\left(4 c^{2}\left(v^{2}-c^{2}\right)^{2}+4 c^{2}\left(3 c^{2}+5 v^{2}\right) \tilde{\eta}+\left(v^{2}+12 c^{2}\right)\left(\tilde{\eta}^{2}-\tilde{\xi}^{2}\right)+4\left(\tilde{\eta}^{3}-3 \tilde{\eta} \tilde{\xi}^{2}\right)\right) \\
& -27 \frac{s^{2}}{\varepsilon^{4} v^{4}}\left(4 c^{2}\left(3 c^{2}+5 v^{2}\right)+2\left(v^{2}+12 c^{2}\right) \tilde{\eta}+4\left(3 \tilde{\eta}^{2}-\tilde{\xi}^{2}\right)\right) \tilde{\xi} i
\end{aligned}
$$

The imaginary part of $\Upsilon$ can be cancelled either by choosing $\tilde{\xi}=0$ or by choosing $\tilde{\xi}^{2}=c^{2}\left(3 c^{2}+5 v^{2}\right)+\left(v^{2}+12 c^{2}\right) \tilde{\eta} / 2+3 \tilde{\eta}^{2}$. In both these cases the real part of $\Upsilon$ can only be cancelled if $\tilde{\eta}<0$. The only exception is if $s=0$, then a multiple root is possible. This case is considered next.

\section{A.1 The $s=0$ case}

For $s=0$ the polynomial in (6) becomes $q(\kappa, 0)=\left(v^{2}-c^{2}\right) \kappa^{2}-\varepsilon v \kappa^{3}$, and has roots

$$
\begin{array}{llll}
v<c: & \kappa_{1}=\frac{v^{2}-c^{2}}{\varepsilon v}, & \kappa_{2}=0, & \kappa_{3}=0 \\
v=c: & \kappa_{1}=0, & \kappa_{2}=0, & \kappa_{3}=0 \\
v>c: & \kappa_{1}=0, & \kappa_{2}=0, & \kappa_{3}=\frac{v^{2}-c^{2}}{\varepsilon v} .
\end{array}
$$


If $v \neq c$, we can find two linearly independent eigenvectors corresponding to the double root $\kappa=0$, so in this case the single root ansatz $\bar{U}_{h}=e^{\kappa x} \Psi$ still holds. When $v=c$, $\kappa=0$ is a triple root, but we can only find two linearly independent eigenvectors. Hence the single root ansatz is no longer valid in this case.

This can also be seen from the $s \rightarrow 0$ limit of $\Phi_{i}^{T} \Psi_{i}$ (the diagonal entries of $E(s)$ in Proposition 4.1). Under the assumption $|s|<<1$ the approximate values of $\kappa_{j}$ and $\Phi_{i}^{T} \Psi_{i}$ can be computed. For $v<c$ we get

$$
\begin{aligned}
& \kappa_{1} \approx \frac{v^{2}-c^{2}}{\varepsilon v}, \quad \kappa_{2} \approx \frac{-s}{v+c}, \quad \kappa_{3} \approx \frac{-s}{v-c} \\
& \Phi_{1}^{T} \Psi_{1} \approx v^{2}-c^{2}, \quad \Phi_{2}^{T} \Psi_{2} \approx 2 c(v+c), \quad \Phi_{3}^{T} \Psi_{3} \approx-2 c(v-c),
\end{aligned}
$$

and for $v>c$ we have the same relations, except that the expression for $\kappa_{1}$ has become $\kappa_{3}$, and vice versa. In both these cases we see that $\Phi_{i}^{T} \Psi_{i} \neq 0$ and hence $E(0)$ is nonsingular. However, the above approximations only hold for $v \neq c$. For $v=c$ the $\kappa_{j}$ 's and $\Phi_{i}^{T} \Psi_{i}$ 's are

$$
\begin{aligned}
& \kappa_{1} \approx-\sqrt{\frac{2 s}{\varepsilon}}, \\
& \kappa_{2} \approx \frac{-s}{2 c}, \\
& \kappa_{3} \approx \sqrt{\frac{2 s}{\varepsilon}} \\
& \Phi_{1}^{T} \Psi_{1} \approx-2 c \sqrt{2 s \varepsilon}, \\
& \Phi_{2}^{T} \Psi_{2} \approx 4 c^{2}, \\
& \Phi_{3}^{T} \Psi_{3} \approx 2 c \sqrt{2 s \varepsilon} .
\end{aligned}
$$

We see that both $\Phi_{1}^{T} \Psi_{1}$ and $\Phi_{3}^{T} \Psi_{3}$ become zero for $s=0$, and consequently the matrix $E(s)$ in Proposition 4.1 is in fact singular for $s=0$ and $v=c$. In this case the ansatz $\bar{U}_{h}=e^{\kappa x} \Psi$ and the general homogeneous solution (7) must be replaced by a double root ansatz. Moreover, new non-reflecting boundary conditions might need to be constructed. In this paper we will simply avoid the special case $v \neq c$.

\section{B The signs of $\operatorname{Re}(\kappa / s)$}

Consider the roots $\kappa_{j}$ of the polynomial $q(\kappa, s)$ in $(6)$. We show that $\operatorname{Re}\left(\kappa_{j} / s\right)$ has the same sign as $\operatorname{Re}\left(\kappa_{j}\right)$ for $j=1,2,3$. Start by denoting $\tilde{\kappa}=\kappa / s$, such that $q(\kappa, s)$ becomes

$$
q(s \tilde{\kappa}, s)=s^{2}\left(1+2 v \tilde{\kappa}+\left(v^{2}-c^{2}-\varepsilon s\right) \tilde{\kappa}^{2}-s \varepsilon v \tilde{\kappa}^{3}\right)=0 .
$$

Let $s=\eta+\xi i$ and assume that $\tilde{\kappa}$ passes the imaginary axis, i.e. $\tilde{\kappa}=\tilde{\beta} i$. Inserting this into $q(s \tilde{\kappa}, s)$ and dividing by $s^{2}$ (assuming $s \neq 0$ ) yields

$$
1-\left(v^{2}-c^{2}-\varepsilon \eta+v \varepsilon \xi \tilde{\beta}\right) \tilde{\beta}^{2}+\left(2 v+\varepsilon \xi \tilde{\beta}+v \varepsilon \eta \tilde{\beta}^{2}\right) \tilde{\beta} i=0 .
$$

There are two options that make the imaginary part of (65) zero. Either we let $\tilde{\beta}=0$ or we let $2 v+\varepsilon \xi \tilde{\beta}+v \varepsilon \eta \tilde{\beta}^{2}=0$. For $\eta \geq 0$, both these choices results in a non-zero real part of $(65)$. We know already that the signs of $\operatorname{Re}\left(\kappa_{j} / s\right)$ are equal to the signs of $\operatorname{Re}\left(\kappa_{j}\right)$ when $s$ is real and positive, and thus $\operatorname{Re}\left(\kappa_{1} / s\right)<0, \operatorname{Re}\left(\kappa_{2} / s\right)<0$ and $\operatorname{Re}\left(\kappa_{3} / s\right)>0$ for all $\operatorname{Re}(s)>0$. 


\section{Proofs of propositions}

\section{C.1 Proof of Proposition 4.3}

Proof. The right boundary condition in (12) yields the solution $\bar{U}_{h}=\sigma_{1} e^{\kappa_{1} x} \Psi_{1}+$ $\sigma_{2} e^{\kappa_{2} x} \Psi_{2}$. Inserting this into $B T_{R}$ in (17) we obtain

$$
B T_{R}=-\left[\begin{array}{l}
\sigma_{1} e^{\kappa_{1} x_{R}} \\
\sigma_{2} e^{\kappa_{2} x_{R}}
\end{array}\right]^{*} \mathcal{A}_{R}\left[\begin{array}{l}
\sigma_{1} e^{\kappa_{1} x_{R}} \\
\sigma_{2} e^{\kappa_{2} x_{R}}
\end{array}\right]
$$

where $\mathcal{A}_{R}$ is a Hermitian matrix as

$$
\mathcal{A}_{R}=\left[\begin{array}{cc}
\Psi_{1}^{*} \widetilde{A} \Psi_{1} & \Psi_{1}^{*} \widetilde{A} \Psi_{2} \\
\Psi_{2}^{*} \widetilde{A} \Psi_{1} & \Psi_{2}^{*} \widetilde{A} \Psi_{2}
\end{array}\right] .
$$

To show that $\mathcal{A}_{R} \geq 0$ we first note that the diagonal elements of $\mathcal{A}_{R}, \Psi_{1}^{*} \widetilde{A} \Psi_{1}$ and $\Psi_{2}^{*} \widetilde{A} \Psi_{2}$, are both positive for $\operatorname{Re}(s)>0$. This is seen from

$$
\Psi_{i}^{*} \widetilde{A} \Psi_{i}=-\operatorname{Re}\left(\frac{s}{\kappa_{i}}\right)\left(c^{2}+\left|\frac{s+v \kappa_{i}}{\kappa_{i}}\right|^{2}\right)-\varepsilon \operatorname{Re}\left(\kappa_{i}\right)\left|\frac{s+v \kappa_{i}}{\kappa_{i}}\right|^{2} \geq 0, \quad \text { for } i=1,2,
$$

where we have used that $\operatorname{Re}\left(\kappa_{i} / s\right)<0$ and $\operatorname{Re}\left(\kappa_{i}\right)<0$ for $i=1,2$. Moreover, the off-diagonal elements $\Psi_{j}^{*} \widetilde{A} \Psi_{i}$ must be sufficiently small, which they are if the quantity $\gamma=\left(\Psi_{1}^{*} \widetilde{A} \Psi_{1}\right)\left(\Psi_{2}^{*} \widetilde{A} \Psi_{2}\right)-\left(\Psi_{1}^{*} \widetilde{A} \Psi_{2}\right)\left(\Psi_{2}^{*} \widetilde{A} \Psi_{1}\right)$ is positive. By using

$\Psi_{j}^{*} \widetilde{A} \Psi_{i}=-\frac{1}{2}\left(\frac{s}{\kappa_{i}}+\frac{s^{*}}{\kappa_{j}^{*}}\right)\left(c^{2}+\left(\frac{s+v \kappa_{j}}{\kappa_{j}}\right)^{*}\left(\frac{s+v \kappa_{i}}{\kappa_{i}}\right)\right)-\frac{\varepsilon}{2}\left(\kappa_{i}+\kappa_{j}^{*}\right)\left(\frac{s+v \kappa_{j}}{\kappa_{j}}\right)^{*}\left(\frac{s+v \kappa_{i}}{\kappa_{i}}\right)$,

it is possible to write

$$
\gamma=\left(\operatorname{Re}\left(\frac{s \varepsilon}{\kappa_{1} \kappa_{2}}\right)\left(c^{2} v^{2}+\left|\frac{s+v \kappa_{1}}{\kappa_{1}}\right|^{2}\left|\frac{s+v \kappa_{2}}{\kappa_{2}}\right|^{2}\right)+\operatorname{Re}\left(\frac{s^{2}}{\kappa_{1} \kappa_{2}}\right) \frac{c^{2}|s|^{2}}{\left|\kappa_{1} \kappa_{2}\right|^{2}}\right)\left|\kappa_{1}-\kappa_{2}\right|^{2}
$$

where the terms $s \varepsilon /\left(\kappa_{1} \kappa_{2}\right)$ and $s^{2} /\left(\kappa_{1} \kappa_{2}\right)$ have positive real parts. This is realized by using the relation $\kappa_{1} \kappa_{2} \kappa_{3}=s^{2} /(\varepsilon v)$ in (9), which leads to

$$
\frac{s \varepsilon}{\kappa_{1} \kappa_{2}}=\varepsilon^{2} v \frac{\kappa_{3}}{s}, \quad \frac{s^{2}}{\kappa_{1} \kappa_{2}}=\varepsilon v \kappa_{3}
$$

where $\operatorname{Re}\left(\kappa_{3} / s\right) \geq 0$ and $\operatorname{Re}\left(\kappa_{3}\right) \geq 0$ according to Proposition 3.1 and Proposition 3.2. Hence $\gamma \geq 0$ and consequently $\mathcal{A}_{R}$ is positive definite and $B T_{R} \leq 0$.

\section{C.2 Proof of Proposition 6.1.}

Proof. Written out explicitly, the matrix $M_{L}=A+\widetilde{H}_{L}+\widetilde{H}_{L}^{T}$ in (34) is

$$
M_{L}=\left[\begin{array}{cc}
v+a \Delta_{\alpha}+a^{*} \Delta_{\alpha}^{*} & c+\alpha_{2}^{*}+b^{*} \Delta_{\alpha}^{*}+a \Delta_{\beta} \\
c+\alpha_{2}+b \Delta_{\alpha}+a^{*} \Delta_{\beta}^{*} & v+\beta_{2}+\beta_{2}^{*}+b \Delta_{\beta}+b^{*} \Delta_{\beta}^{*}
\end{array}\right],
$$


where the choice of $a$ and $b$ affects the eigenvalues of $M_{L}$. For $M_{L}$ to be negative semi-definite it is needed that either $(i)$ or $(i i)$ holds, together with $($ iii $)$.

$$
\begin{array}{ll}
\text { i) } & v+2 \operatorname{Re}\left(a \Delta_{\alpha}\right) \leq 0 \\
\text { ii) } & v+2 \operatorname{Re}\left(\beta_{2}+b \Delta_{\beta}\right) \leq 0 \\
\text { iii }) & \left(v+2 \operatorname{Re}\left(a \Delta_{\alpha}\right)\right)\left(v+2 \operatorname{Re}\left(b \Delta_{\beta}+\beta_{2}\right)\right)-\left|c+b \Delta_{\alpha}+\alpha_{2}+a^{*} \Delta_{\beta}^{*}\right|^{2} \geq 0 .
\end{array}
$$

The requirement ( iii) leads to the equivalent relation (35), which we identify as the equation for a parabola with complex coefficients in the $x=a \Delta_{\beta}^{*}-b \Delta_{\alpha}^{*}, y=a \Delta_{\alpha}+b \Delta_{\beta}$ coordinate system. Further, we note that $r$ in (36) is negative, by rewriting it as

$$
r=-\frac{v|s|^{2}+v\left|\kappa_{3}\right|^{2} \operatorname{Re}\left(s / \kappa_{3}\right)^{2}+\left(v^{2}\left|\kappa_{3}\right|^{2}+|s|^{2}\right) \operatorname{Re}\left(s / \kappa_{3}\right)+\frac{1}{2} v \varepsilon\left|\kappa_{3}\right|^{2} \operatorname{Re}\left(s+v \kappa_{3}\right)}{2\left|v \kappa_{3}+s\right|^{2}} \leq 0
$$

where we have used that $\operatorname{Re}\left(\kappa_{3}\right) \geq 0$ and $\operatorname{Re}\left(\kappa_{3} / s\right) \geq 0$. Then, by rearranging equation (35) as

$$
\begin{aligned}
& v+2 \operatorname{Re}\left(a \Delta_{\alpha}\right) \leq \frac{1}{r}\left|a \Delta_{\beta}^{*}-b \Delta_{\alpha}^{*}+\frac{v \Delta_{\beta}^{*}-\left(c+\alpha_{2}\right) \Delta_{\alpha}^{*}}{\Delta_{\alpha}}\right|^{2} \leq 0 \\
& v+2 \operatorname{Re}\left(\beta_{2}+b \Delta_{\beta}\right) \leq \frac{\left|\Delta_{\beta}\right|^{2}}{r\left|\Delta_{\alpha}\right|^{2}}\left|a \Delta_{\beta}^{*}-b \Delta_{\alpha}^{*}-\frac{\left(v+2 \operatorname{Re}\left(\beta_{2}\right)\right) \Delta_{\alpha}^{*}-\left(c+\alpha_{2}^{*}\right) \Delta_{\beta}^{*}}{\Delta_{\beta}}\right|^{2} \leq 0,
\end{aligned}
$$

we see that if $($ iii $)$ holds, so do the relations $(i)$ and $(i i)$. Hence the pairs of $a$ and $b$ fulfilling (35) lead to negative semi-definiteness of $M_{L}$.

\section{C.3 Proof of Proposition 6.6}

Proof. Using that $\widetilde{A}=x_{+} \Lambda_{+} x_{+}^{T}+x_{-} \Lambda_{-} x_{-}^{T}$ and denoting the components of $\mathcal{A}_{R}$ in (19) as $\mathcal{A}_{R}^{j i}$, gives

$$
\begin{aligned}
\mathcal{A}_{R}^{j i} & =\Psi_{j}^{*} \widetilde{A} \Psi_{i} \\
& =\Psi_{j}^{*}\left(x_{+} \Lambda_{+} x_{+}^{T}+x_{+} R_{R}^{*} \Lambda_{-} R_{R} x_{+}^{T}\right) \Psi_{i}+\Psi_{j}^{*}\left(x_{-} \Lambda_{-} x_{-}^{T}-x_{+} R_{R}^{*} \Lambda_{-} R_{R} x_{+}^{T}\right) \Psi_{i} \\
& =\Psi_{j}^{*} x_{+} \mathcal{C}_{R} x_{+}^{T} \Psi_{i}+\underbrace{\Psi_{j}^{*}\left(x_{-} \Lambda_{-} x_{-}^{T}-x_{+} \bar{R}_{R}^{*} \Lambda_{-} \bar{R}_{R} x_{+}^{T}\right) \Psi_{i}}_{\mathcal{N}_{R}^{j i}} .
\end{aligned}
$$

Inserting the relation $x_{-}^{T}+R_{R} x_{+}^{T}=J_{R}^{-1} \bar{L}_{R}$ from (43) into $\mathcal{N}_{L}$ we obtain

$$
\begin{aligned}
\mathcal{N}_{R}^{j i} & =\Psi_{j}^{*}\left(x_{-} \widetilde{\Lambda}_{-} x_{-}^{T}-x_{+} R_{R}^{*} \widetilde{\Lambda}_{-} R_{R} x_{+}^{T}\right) \Psi_{i} \\
& =\frac{1}{2} \Psi_{j}^{*}\left(\left(x_{-}^{T}+R_{R} x_{+}^{T}\right)^{*} \Lambda_{-}\left(x_{-}^{T}-R_{R} x_{+}^{T}\right)+\left(x_{-}^{T}-R_{R} x_{+}^{T}\right)^{*} \Lambda_{-}\left(x_{-}^{T}+R_{R} x_{+}^{T}\right)\right) \Psi_{i} \\
{[\text { use (43)] }} & =\frac{1}{2} \Psi_{j}^{*}\left(\left(J_{R}^{-1} \bar{L}_{R}\right)^{*} \Lambda_{-}\left(x_{-}^{T}-R_{R} x_{+}^{T}\right)+\left(x_{-}^{T}-R_{R} x_{+}^{T}\right)^{*} \Lambda_{-} J_{R}^{-1} \bar{L}_{R}\right) \Psi_{i} \\
& =\left(\bar{L}_{R} \Psi_{j}\right)^{*} \frac{\left(J_{R}^{-1}\right)^{*} \Lambda_{-}\left(x_{-}^{T}-R_{R} x_{+}^{T}\right) \Psi_{i}}{2}+\frac{\Psi_{j}^{*}\left(x_{-}^{T}-R_{R} x_{+}^{T}\right)^{*} \Lambda_{-} J_{R}^{-1}}{2} \bar{L}_{R} \Psi_{i} .
\end{aligned}
$$


Recall that $\bar{L}_{R}=\Phi_{3}^{T}$ and that $\Phi_{j}^{T} \Psi_{i}=0$. This makes $\bar{L}_{R} \Psi_{1}=0$ and $\bar{L}_{R} \Psi_{2}=0$. Thus $\mathcal{N}_{R}^{j i}=0$ for all $i=1,2$ and $j=1,2$, and we get

$$
\mathcal{A}_{R}^{j i}=\Psi_{j}^{*} x_{+} \mathcal{C}_{R} x_{+}^{T} \Psi_{i}, \quad i=1,2, \quad j=1,2 .
$$

From these coefficients we compose

$$
\mathcal{A}_{R}=\left(x_{+}^{T}\left[\begin{array}{ll}
\Psi_{1} & \Psi_{2}
\end{array}\right]\right)^{*} \mathcal{C}_{R} x_{+}^{T}\left[\begin{array}{ll}
\Psi_{1} & \Psi_{2}
\end{array}\right]
$$

where $x_{+}^{T}\left[\Psi_{1} \Psi_{2}\right]$ is a non-singular $2 \times 2$ matrix. Since $\mathcal{A}_{R} \geq 0$ from Proposition 4.3 , we know that $\mathcal{C}_{R} \geq 0$.

\section{Penalty parameters}

\section{D.1 Dirichlet boundary conditions}

Giving Dirichlet boundary conditions implies that $L_{L}=I_{2}$ and $L_{R}=[0,1]$ in the numerical scheme $(1)$, and that the terms $\left(\left(\Sigma_{0, N} * V\right)(t)-\Gamma_{0, N}\right)$ in $(23)$ are replaced by $\left(P^{-1} e_{0, N} \otimes \tau_{0, N}^{D i r}+P^{-1} D^{T} e_{0, N} \otimes \sigma_{0, N}^{D i r}\right)\left(L_{L, R} V_{0, N}-g_{L, R}\right)$. If the Dirichlet boundary conditions are used at both boundaries, the scheme becomes

$$
\begin{aligned}
V_{t}+\left(D_{1} \otimes A\right) V-\left(D_{2} \otimes B\right) V & =\left(P^{-1} e_{0} \otimes \tau_{0}^{D i r .}+P^{-1} D^{T} e_{0} \otimes \sigma_{0}^{D i r .}\right)\left(L_{L} V_{0}-g_{L}\right) \\
& +\left(P^{-1} e_{N} \otimes \tau_{N}^{D i r .}+P^{-1} D^{T} e_{N} \otimes \sigma_{N}^{D i r .}\right)\left(L_{R} V_{N}-g_{R}\right) .
\end{aligned}
$$

By choosing the penalty matrices as

$$
\tau_{0}^{\text {Dir. }}=-\left[\begin{array}{ll}
v & c \\
v & c
\end{array}\right], \quad \sigma_{0}^{\text {Dir. }}=\left[\begin{array}{ll}
0 & 0 \\
0 & \varepsilon
\end{array}\right], \quad \tau_{N}^{\text {Dir. }}=\left[\begin{array}{c}
c-v \\
v-c
\end{array}\right], \quad \sigma_{N}^{\text {Dir. }}=-\left[\begin{array}{l}
0 \\
\varepsilon
\end{array}\right],
$$

the scheme becomes stable for $0<v<c$.

\section{D.2 Approximate non-reflecting boundary conditions}

The approximative boundary conditions are derived by inserting $s=0$ into the exact non-reflecting boundary operators, which yields time-local, low-reflecting boundary conditions. They are expressed as $H_{L, R}^{\text {low }} U+G_{L, R}^{\text {low }} U_{x}=g_{L, R}$, where

$$
\begin{aligned}
H_{L}^{\text {low }} & =-\left[\begin{array}{cc}
0 & 0 \\
v+c & v+c
\end{array}\right] & G_{L}^{\text {low }} & =\left[\begin{array}{ll}
0 & \varepsilon \\
0 & \varepsilon
\end{array}\right] \\
H_{R}^{\text {low }} & =\left[\begin{array}{cc}
v-c & c-v
\end{array}\right], & G_{R}^{\text {low }} & =\left[\begin{array}{ll}
0 & \varepsilon
\end{array}\right],
\end{aligned}
$$

which is $H_{L, R}$ and $G_{L, R}$ in (22), evaluated at $s=0$ and for $0<v<c$. If the approximate NRBC's are imposed at both boundaries, the numerical scheme (23) becomes

$$
\begin{aligned}
V_{t}+\left(D_{1} \otimes A\right) V-\left(D_{2} \otimes B\right) V & =\left(P^{-1} e_{0} \otimes \tau_{0}^{\text {low }}+P^{-1} D^{T} e_{0} \otimes \sigma_{0}^{\text {low }}\right)\left(H_{L}^{\text {low }} V_{0}+G_{L}^{\text {low }}(\bar{D} V)_{0}\right) \\
& +\left(P^{-1} e_{N} \otimes \tau_{N}^{\text {low }}+P^{-1} D^{T} e_{N} \otimes \sigma_{N}^{\text {low }}\right)\left(H_{R}^{\text {low }} V_{N}+G_{R}^{\text {low }}(\bar{D} V)_{N}\right),
\end{aligned}
$$


where we have used $g_{L, R}=0$. Following the "replacing the indefinite terms" approach, by evaluating the penalty (59) at $s=0$, we obtain

$$
\tau_{0}^{l o w}=S_{L}, \quad \sigma_{0}^{l o w}=\left[\begin{array}{ll}
0 & 0 \\
0 & 0
\end{array}\right], \quad \tau_{N}^{l o w}=-S_{R}, \quad \sigma_{N}^{l o w}=\left[\begin{array}{l}
0 \\
0
\end{array}\right] .
$$

In $S_{L}$ the constants $a, b$ must be chosen properly, correspondingly to Proposition 6.1. Instead following the "replacing the ingoing variables" approach, by evaluating the penalty $(60)$ at $s=0$, yields

$$
\tau_{0}^{\text {low }}=\left[\begin{array}{ll}
0 & 1 / 2 \\
0 & 1 / 2
\end{array}\right], \quad \sigma_{0}^{\text {low }}=\left[\begin{array}{cc}
0 & 0 \\
\frac{\varepsilon v}{v^{2}-c^{2}} & \frac{\varepsilon}{2(v+c)}
\end{array}\right], \quad \tau_{N}^{\text {low }}=\left[\begin{array}{c}
1 / 2 \\
-1 / 2
\end{array}\right], \quad \sigma_{N}^{\text {low }}=\left[\begin{array}{c}
0 \\
\frac{\varepsilon}{2(v-c)}
\end{array}\right]
$$

for $0<v<c$.

\section{References}

[1] D. Appelö, T. Hagstrom, and G. Kreiss. Perfectly matched layers for hyperbolic systems: general formulation, well-posedness, and stability. SIAM J. Appl. Math., $67(1): 1-23,2006$.

[2] E. Bécache, D. Givoli, and T. Hagstrom. High-order absorbing boundary conditions for anisotropic and convective wave equations. Journal of Computational Physics, 229(4):1099-1129, 2010.

[3] J.P. Berenger. A perfectly matched layer for the absorption of electromagnetic waves. Journal of computational physics, 114(2):185-200, 1994.

[4] M. H. Carpenter, D. Gottlieb, and S. Abarbanel. Time-stable boundary conditions for finite-difference schemes solving hyperbolic systems: Methodology and application to high-order compact schemes. Journal of Computational Physics, 111(2):220-236, 1994.

[5] M.H. Carpenter, J. Nordström, and D. Gottlieb. A stable and conservative interface treatment of arbitrary spatial accuracy. Journal of Computational Physics, 148:341-365, 1999.

[6] B. Engquist and B. Gustafsson. Steady state computations for wave propagation problems. Mathematics of Computations, 49:39-64, 1987.

[7] B. Engquist and A. Majda. Absorbing boundary conditions for the numerical simulation of waves. Mathematics of Computation, 31(139):629-651, 1977.

[8] L. Ferm. Non-reflecting boundary conditions for the steady Euler equations. Journal of Computational Physics, 122:307-316, 1995.

[9] M. Grote and J. Keller. Exact nonreflecting boundary conditions for the time dependent wave equation. SIAM Journal on Applied Mathematics, 55(2):280-297, 1995. 
[10] M. J. Grote and J. B. Keller. Nonreflecting boundary conditions for time-dependent scattering. Journal of Computational Physics, 127(1):52-65, 1996.

[11] B. Gustafsson. Far-field boundary conditions for time-dependent hyperbolic systems. SIAM J. ScI. Statist. Comput., 9(5):812-828, 1988.

[12] B. Gustafsson and H.-O. Kreiss. Boundary conditions for time dependent problems with an artificial boundary. Journal of Computational Physics, 30(3):333-351, 1979 .

[13] B. Gustafsson, H.-O. Kreiss, and J. Oliger. Time Dependent Problems and Difference Methods. John Wiley \& Sons, Inc., 1995.

[14] B. Gustafsson, H.-O. Kreiss, and A. Sundström. Stability theory of difference approximations for mixed initial boundary value problems. II. Mathematics of Computation, 26(119):649-686, 1972.

[15] T. Hagstrom. Radiation boundary conditions for the numerical simulation of waves. Acta Numerica, 8:47-106, 1999.

[16] T. Hagstrom, E. Bécache, D. Givoli, and K. Stein. Complete radiation boundary conditions for convective waves. Commun. Comput. Phys., 11(2):610-628, 2012.

[17] L. Halpern. Artificial boundary conditions for incompletely parabolic perturbations of hyperbolic systems. SIAM Journal on Mathematical Analysis, 22(5):1256-1283, 1991.

[18] J.S. Hesthaven. On the analysis and construction of perfectly matched layers for the linearized euler equations. Journal of Computational Physics, 142(1):129-147, 1998.

[19] J.E. Hicken and D.W. Zingg. Summation-by-parts operators and high-order quadrature. Journal of Computational and Applied Mathematics, 237(1):111-125, 2013.

[20] C. Lubich. Convolution quadrature and discretized operational calculus. I. Numerische Mathematik, 52:129-145, 1988.

[21] C. Lubich. Convolution quadrature and discretized operational calculus. II. Numerische Mathematik, 52:413-425, 1988.

[22] C. Lubich and A. Schädle. Fast convolution for nonreflecting boundary conditions. SIAM J. Sci. Comput., 24(1):161-182, 2002.

[23] K. Mattsson. Boundary procedures for summation-by-parts operators. Journal of Scientific Computing, 18(1):133-153, 2003.

[24] J. Nordström. The influence of open boundary conditions on the convergence to steady state for the Navier-Stokes equations. Journal of Computational Physics, 85:210-244, 1989. 
[25] J. Nordström and M. H. Carpenter. Boundary and interface conditions for high order finite difference methods applied to the Euler and Navier-Stokes equations. Journal of Computational Physics, 148:621-645, 1999.

[26] J. Nordström, S. Eriksson, and P. Eliasson. Weak and strong wall boundary procedures and convergence to steady-state of the Navier-Stokes equations. Journal of Computational Physics, 231(14):4867-4884, 2012.

[27] J. Nordström, J. Gong, E. van der Weide, and M. Svärd. A stable and conservative high order multi-block method for the compressible Navier-Stokes equations. Journal of Computational Physics, 228(24):9020-9035, 2009.

[28] L. Råde and B. Westergren. Mathematics Handbook for Science and Engineering. Studentlitteratur, Lund, 1998.

[29] I. L. Sofronov. Non-reflecting inflow and outflow in a wind tunnel for transonic time-accurate simulation. Journal of Mathematical Analysis and Applications, 221(1):92-115, 1998.

[30] J. C. Strikwerda. Initial boundary value problems for incompletely parabolic systems. Communications on Pure and Applied Mathematics, 30(6):797-822, 1977.

[31] M. Svärd, M.H. Carpenter, and J. Nordström. A stable high-order finite difference scheme for the compressible Navier-Stokes equations: far-field boundary conditions. Journal of Computational Physics, 225(1):1020-1038, 2007.

[32] S. V. Tsynkov. Numerical solution of problems on unbounded domains. A review. Appl. Numer. Math., 27:465-532, August 1998. 